\title{
Solar wind alpha particles and heavy ions in the inner heliosphere observed with MESSENGER
}

\author{
Daniel J. Gershman, ${ }^{1}$ Thomas H. Zurbuchen, ${ }^{1}$ Lennard A. Fisk, ${ }^{1}$ Jason A. Gilbert, ${ }^{1}$ \\ Jim M. Raines, ${ }^{1}$ Brian J. Anderson, ${ }^{2}$ Charles W. Smith, ${ }^{3}$ Haje Korth, ${ }^{2}$ \\ and Sean C. Solomon ${ }^{4,5}$ \\ Received 12 April 2012; revised 28 June 2012; accepted 10 July 2012; published 15 September 2012.
}

[1] The Fast Imaging Plasma Spectrometer (FIPS) on the MErcury Surface, Space ENvironment, GEochemistry, and Ranging (MESSENGER) spacecraft has made the first in situ measurements of solar wind plasma in the inner heliosphere since the Helios 1 and 2 spacecraft in the 1980s. Although the core of the solar wind velocity distribution is obstructed by the spacecraft sunshade, a data analysis technique has been developed that recovers both bulk and thermal speeds to $10 \%$ accuracy and provides the first measurements of solar wind heavy ions (mass per charge $>2 \mathrm{amu} / \mathrm{e}$ ) at heliocentric distances within 0.5 AU. Solar wind alpha particles and heavy ions appear to have similar mean flow speeds at values greater than that of the protons by approximately $70 \%$ of the Alfvén speed. From an examination of the thermal properties of alpha particles and heavier solar wind ions, we find a ratio of the temperature of alpha particles to that of protons nearly twice that of previously reported Helios observations, though still within the limits of excessive heating of heavy ions observed spectroscopically close to the Sun. Furthermore, examination of typical magnetic power spectra at the orbits of MESSENGER and at 1 AU reveals the lack of a strong signature of local resonant ion heating, implying that a majority of heavy ion heating could occur close to the Sun. These results demonstrate that the solar wind at $\sim 0.3 \mathrm{AU}$ is a blend of the effects of wave-particle interactions occurring in both the solar corona and the heliosphere.

Citation: Gershman, D. J., T. H. Zurbuchen, L. A. Fisk, J. A. Gilbert, J. M. Raines, B. J. Anderson, C. W. Smith, H. Korth, and S. C. Solomon (2012), Solar wind alpha particles and heavy ions in the inner heliosphere observed with MESSENGER, J. Geophys. Res., 117, A00M02, doi:10.1029/2012JA017829.

\section{Introduction}

[2] The solar wind is the manifestation of the ubiquitous expansion of the hot solar corona into space. Although the origin, heating, and acceleration of the solar wind are still much debated, there is consensus on a number of key issues. Initial models of the solar wind were based on the assumption of an isothermal plasma, with electrons and ions having equal temperatures [Parker, 1958]. However, we know from

\footnotetext{
${ }^{1}$ Department of Atmospheric, Oceanic and Space Sciences, University of Michigan, Ann Arbor, Michigan, USA.

${ }^{2}$ The Johns Hopkins University Applied Physics Laboratory, Laurel, Maryland, USA.

${ }^{3}$ Physics Department, Space Science Center, University of New Hampshire, Durham, New Hampshire, USA.

${ }^{4}$ Department of Terrestrial Magnetism, Carnegie Institution of Washington, Washington, D. C., USA.

${ }^{5}$ Lamont-Doherty Earth Observatory, Columbia University, Palisades, New York, USA.

Corresponding author: D. J. Gershman, Department of Atmospheric, Oceanic and Space Sciences, University of Michigan, Ann Arbor, MI 48105, USA. (djgersh@umich.edu)

C2012. American Geophysical Union. All Rights Reserved. 0148-0227/12/2012JA017829
}

ultraviolet observations of neutral hydrogen coupled to solar wind plasmas near the Sun through charge exchange and from direct observations of heavy ions (mass $>4 \mathrm{amu}$ ) that the ions are preferentially heated, and that this heating is necessary to attain the observed solar wind speeds [Kohl et al., 1997, 1998]. At about 2 solar radii, solar wind ions associated with coronal holes have relative temperatures that strongly depend on the ratio of their mass to their charge $(\mathrm{m} / \mathrm{q})$; for instance, ratios of the temperatures of $\mathrm{Mg}^{9+}$ and $\mathrm{O}^{5+}$ to that of neutral hydrogen, $T_{\mathrm{Mg}^{9+}} / T_{\mathrm{H}}$ and $T_{\mathrm{O}^{5+}} / T_{\mathrm{H}}$, are approximately equal to 64 at 1.7 and 2.2 solar radii, respectively [Kohl et al., 1997]. Similarly, the derived flow speeds near 2 solar radii are also higher for heavy ions, with $v_{\mathrm{O}^{5+}} /$ $v_{\mathrm{H}} \sim 2$ [Kohl et al., 1998].

[3] These remote sensing data elucidate the important role of wave-particle interactions in most parts of the closed and open corona, even though the precise physical processes involved in these interactions is still debated [e.g., Fisk et al., 1999; Verdini and Velli, 2007; Cranmer et al., 2007]. Most importantly, these data show that the collisional coupling of heavy ions to solar wind protons is not as dominant as initially assumed in the first comprehensive model of heavy ions by Geiss et al. [1970]. In these collision-dominated models, heavy ions are coupled to the accelerating protons 
through Coulomb collisions, and thus their velocity is typically smaller than that of protons. Similarly, due to the dominance of these collisions, heavy ions were predicted to have thermal speeds smaller than those of protons, again in contradiction with key results from the Ultraviolet Coronograph Spectrometer (UVCS) [Kohl et al., 1995] on the Solar and Heliospheric Observatory (SOHO).

[4] In situ observations of solar wind heavy ions to date have been limited to heliocentric distances of $1 \mathrm{AU}$ and beyond. These measurements were performed mostly by solar wind composition instruments such as those on the Advanced Composition Explorer (ACE), Ulysses, and Solar TErrestrial RElations Observatory (STEREO) spacecraft [Gloeckler et al., 1992, 1998; Galvin et al., 2008]. Through their filtering of ions by energy per charge $(E / q)$ and the measurement, after post-acceleration, of time of flight and total energy, the velocity $(v)$, mass $(m)$, and charge $(q)$ of heavy ions are determined. There are some observational limitations that are worth mentioning: the Solar Wind Ion Composition Spectrometer (SWICS) instruments on ACE and Ulysses measure only the speed of each ion and do not have sufficient angular resolution to recover the full threedimensional orientation of the velocity vector, prohibiting the analysis of full distribution functions [see von Steiger and Zurbuchen, 2006]. The Plasma and Suprathermal Ion Composition (PLASTIC) instrument on STEREO, however, has the ability to resolve incident direction [Bochsler et al., 2010].

[5] Generally, these observations near 1 AU show a streaming of heavy ions that is faster than protons along the magnetic field at differential velocities that are of the same order, but typically less than the Alfvén speed [Asbridge et al., 1976; Zurbuchen et al., 2000; Berger et al., 2011]. The actual amount of differential streaming depends on the mass and charge of ions in a way that is not fully understood [Hefti et al., 1998; von Steiger and Zurbuchen, 2006], although there seems to be an ordering of the data by $q^{2} / \mathrm{m}$, indicating a role of collisional effects. Heavy ions observed near 1 AU also tend to have thermal speeds that are approximately equal, indicating a mass-proportional temperature. From a comparison of data gathered near $1 \mathrm{AU}$ with data from Ulysses that span up to $5.4 \mathrm{AU}$, it is evident that, with increasing heliocentric distance, the solar wind becomes increasingly less collisional.

[6] Simple electrostatic analysis can be used for the measurement of the kinetic properties of alpha particles relative to protons during time periods when the solar wind is not too hot [Neugebauer, 1976; Marsch et al., 1982a]. Such analyses are used as part of the Solar Wind Electron, Proton and Alpha Monitor (SWEPAM) [McComas et al., 1998a] or Faraday cup [e.g., Ogilvie et al., 1995] experiments. These works provide an intriguing picture of the importance of collisional processes, wave-particle interactions, and plasma instabilities. In interpreting these data, it is critical to recognize that the alpha particles carry $\sim 20 \%$ of the internal pressure of a solar wind plasma [McKenzie et al., 1995] and are therefore a major contributor to plasma dynamics, such as the dispersion properties of waves and instabilities [e.g., Gary et al., 2001]. Following a long-duration analysis of alpha particle and proton data near $1 \mathrm{AU}$, Kasper et al. [2008] demonstrated the importance of collisional processes that shape the relative speed, temperature, and temperature anisotropies of alpha particles and protons. They also argued that there is local heating through the local dissipation of kinetic Alfvén waves.

[7] The differential heating of heavy ions with respect to the protons is expected to occur near the transition of two subintervals of the magnetic field spectra: the inertial range and the dissipation range [Coleman, 1968; Bavassano et al., 1982; Frisch, 1996]. The inertial range as it is expected to exist in the plasma frame occurs at frequencies substantially lower than the gyrofrequency of heavy ions, obscured by Doppler shifts that are a consequence of measuring the magnetic field $\mathbf{B}$ in the spacecraft frame. Here, the energy transport occurs in an approximately scale-invariant cascade process, carrying energy to successively smaller spatial scales and higher values of frequency $(f)$ without any dissipation. The spectral slope of this transport varies as $f^{-5 / 3}$ or $f^{-3 / 2}$ for dominant fluid dynamics (Kolmogorov) or magnetic transport effects (Kraichnan), respectively. At sufficiently high frequencies, dissipation sets in [Leamon et al., 1998]. This dissipation strongly depends on the kinetic properties of the plasma. The importance of various dissipation processes is a subject of current debate [Leamon et al., 1998, 1999; Bale et al., 2005; Hamilton et al., 2008; Smith et al., 2012]. In order to test the predictions of such turbulence models, a combined dataset of heavy ions and magnetic field measurements is required at multiple heliocentric distances.

[8] The only comprehensive analysis of solar wind ions in the inner heliosphere was made by the Helios spacecraft in the late 1970s and early 1980s. Helios 1 and Helios 2 had highly eccentric heliospheric orbits with perihelia of $0.29 \mathrm{AU}$ and $0.3 \mathrm{AU}$, respectively. The mission, payload, and plasma instrument were described by Schwenn et al. [1975] and Rosenbauer et al. [1977]. The distribution functions of protons [Marsch et al., 1982b] and alpha particles [Marsch et al., 1982a] were characterized in detail, including examinations of the temperatures and differential flow speeds between alpha particles and protons as functions of heliocentric distance and solar wind speed. However, the Helios payload included no heavy ion instruments, and there have been no new in situ measurements in the inner heliosphere for the past 30 years.

[9] Here, we focus on the dynamic properties of heavy ions measured with a mass-resolving instrument that is part of the MErcury Surface, Space ENvironment, GEochemistry, and Ranging (MESSENGER) spacecraft that is currently in orbit about Mercury [Solomon et al., 2001]. We provide the first combined measurements of solar wind protons, alpha particles, and a group of heavy ions, and we place these data into context with previous observations by Helios and those more recently obtained near 1 AU. Specifically, we address the nonthermal behavior of alpha particles and heavy ions, including a discussion of the typical solar wind turbulent environment inferred from high-resolution magnetic field measurements from both MESSENGER and ACE. In Section 2, we provide an introduction of the methodology for deriving the relevant kinetic parameters from partially obscured measurements and show the recovered solar wind parameters for the inner heliosphere as measured by MESSENGER. In Section 3, we detail observations related to the thermal and kinetic properties of 


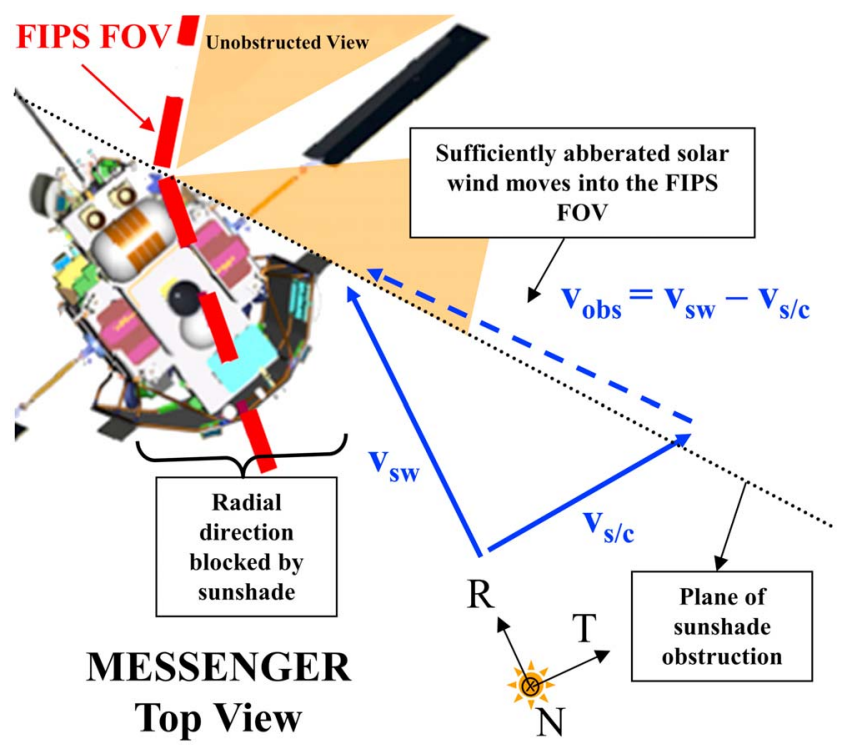

Figure 1. Top view of a portion of the MESSENGER spacecraft depicting the FIPS field of view and the obstruction of the solar direction by the spacecraft sunshade. Because of substantial aberration of $\mathbf{v}_{\mathrm{sw}}$ from the spacecraft velocity $\mathbf{v}_{\mathrm{s} / \mathrm{c}}$, radially traveling particles are measured with an effective velocity $\mathbf{v}_{\mathrm{obs}}=\mathbf{v}_{\mathrm{sw}}-\mathbf{v}_{\mathrm{s} / \mathrm{c}}$ by the spacecraft, allowing, under some conditions, a fraction of the solar wind distribution to be sampled by FIPS. The coordinate $R$ is radial to the Sun, $T$ is formed by the cross product of the solar rotation axis and $R$ and lies in the equatorial plane, and $N$ completes the right-handed system.

alpha particles and heavy ions with respect to the protons. Finally, a concluding discussion of our results appears in Section 4.

\section{Solar Wind Observations by MESSENGER}

[10] The Fast Imaging Plasma Spectrometer (FIPS), one of two charged particle sensors on MESSENGER's Energetic Particle and Plasma Spectrometer (EPPS) instrument [Andrews et al., 2007], was designed to measure magnetospheric ions around Mercury with $m / q$ ratios up to $40 \mathrm{amu} / \mathrm{e}$ and $E / q$ ratios ranging from $40 \mathrm{eV} / \mathrm{e}$ to $10 \mathrm{keV} / \mathrm{e}$, with energy scan times of $64 \mathrm{~s}$ or $8 \mathrm{~s}$ depending on instrument operating mode. FIPS has a near hemispherical instantaneous field of view, with $1.4 \pi \mathrm{sr}$ imaged during each scan at an angular resolution of approximately $10^{\circ}$. Typical solar wind plasmas fall well within these mass and energy ranges, such that FIPS is fully capable of resolving the solar wind, including several of its less abundant ion species and pickup ions. However, as a consequence of the mission's thermal design, the direct line of sight of FIPS in the solar direction is obstructed by the MESSENGER spacecraft sunshade, as illustrated in Figure 1. This obstruction nominally prevents the instrument from sampling the majority of the solar wind distribution. The time period of the observations presented here is during the first year of MESSENGER's primary orbital mission, from 26 March 2011 to 19 December 2011. During this period, MESSENGER's heliocentric distance varied between $0.3 \mathrm{AU}$ and $0.5 \mathrm{AU}$.
[11] Fortunately, due to nonradial particle velocities from magnetic turbulence, increased plasma temperatures close to the Sun, and aberration from a substantial spacecraft velocity in the tangential direction, a portion of the solar wind distribution function can often be sampled by the instrument and analyzed. After deriving the moments of a partial Maxwell-Boltzmann distribution function (Appendix A), we find that whereas it is difficult to calculate accurately the plasma density without additional constraints on the threedimensional solar wind bulk velocity, the solar wind speed and thermal velocity should be readily recoverable. Convected isotropic Maxwell-Boltzmann velocity distributions were used in these derivations to enable near-analytical integrations, in recognition of the fact that these are idealized distributions. The derived moments are then applied to more realistic distributions such as those that include a strong suprathermal tail and temperature anisotropy. This section describes a methodology for recovering estimates of solar wind speed and thermal velocities of diverse ion species from such partial distributions, as well as an analysis of the corresponding recovery errors.

\subsection{Distribution Functions for Instrument Observations}

[12] A coordinate system in the FIPS instrument frame with respect to a partially observed solar wind plasma is defined in Figure 2. This coordinate system will enable the derivation of a distribution function for FIPS observations that can be used to recover solar wind bulk and thermal speeds. The spacecraft sunshade blocks all incident particles angles within about $12^{\circ}$ of the radial direction. The edge of the sunshade in the spacecraft ram direction, within the FIPS $\sim 1.4 \pi$ sr field of view (FOV), defines a plane on one side of which solar wind particles may enter the instrument aperture and on the other side of which they are obstructed (Figure 1). The $z$-axis is defined to be perpendicular to this plane, such that the $+z$ direction points away from the obstruction. The $x-y$ plane, therefore, is the plane through the end of this obstruction. The average velocity of the solar wind is denoted $v_{\mathrm{o}}$, and its direction is defined such that the center of the distribution lies in the $x-z$ plane with coordinates

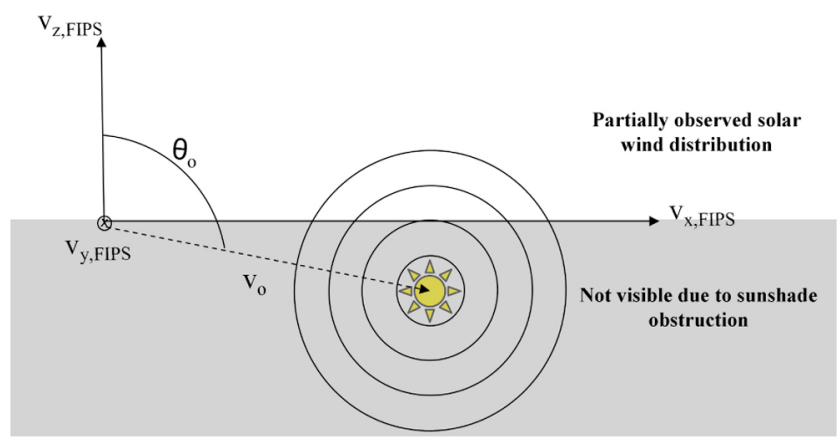

Figure 2. Coordinate system centered in the MESSENGER FIPS frame. Here, the $+z$-axis points away from the obstruction defined by the edge of the sunshade in the FIPS FOV, and the solar wind core is centered at $\left[v_{\mathrm{o}} \sin \left(\theta_{\mathrm{o}}\right), 0\right.$, $\left.v_{\mathrm{o}} \cos \left(\theta_{\mathrm{o}}\right)\right]$. Only the portion of the solar wind distribution in the unshaded area is measured by FIPS. 
$\left[v_{\mathrm{o}} \sin \left(\theta_{\mathrm{o}}\right), 0, v_{\mathrm{o}} \cos \left(\theta_{\mathrm{o}}\right)\right]$, i.e. $\phi_{\mathrm{o}}=0$. The angle $\left|\pi / 2-\theta_{\mathrm{o}}\right|$ represents the minimum angular distance from the distribution center that lies within the instrument's FOV. Given the sunshade blockage noted above and an aberration provided by the spacecraft velocity up to $7^{\circ}, \theta_{\mathrm{o}} \gtrsim 5^{\circ}$ for a purely radially flowing solar wind. This angle can be further reduced via the turbulent motions of the solar wind plasmas. Following standard spherical coordinate system definitions, $\theta$ is the angle from the $z$-axis and $\phi$ is the clock angle in the $x-y$ plane.

[13] We assume that any other obstructions to the instrument FOV are negligible, so that the solar wind distribution is visible over the ranges $\theta=[0, \pi / 2]$ and $\phi=[0,2 \pi]$. The highly supersonic solar wind is confined to a small angular width in the instrument FOV, so other obstructions, such as solar array panels or small protrusions of the spacecraft body into the FOV, should not markedly affect the fraction of the distribution observed.

\subsubsection{Isotropic Maxwell-Boltzmann Distributions}

[14] With the newly defined coordinate system from above, a model for the solar wind distribution function takes the form of an isotropic drifting Maxwell-Boltzmann distribution in spherical coordinates:

$$
\begin{aligned}
& f(v, \theta, \phi)=n_{\mathrm{o}}\left(\frac{1}{2 \pi}\right)^{3 / 2} \frac{1}{v_{\mathrm{th}}^{3}} \\
& \cdot \exp \left(-\frac{v^{2}+v_{\mathrm{o}}^{2}-2 v v_{\mathrm{o}}\left(\sin \theta \sin \theta_{\mathrm{o}} \cos \phi+\cos \theta \cos \theta_{\mathrm{o}}\right)}{2 v_{\mathrm{th}}^{2}}\right),
\end{aligned}
$$

where $n_{\mathrm{o}}$ is the plasma density and $v_{\text {th }}$ is related to the temperature $T$ by $v_{\text {th }} \equiv \sqrt{k_{\mathrm{B}} T / m}$ where $k_{\mathrm{B}}$ is the Boltzmann constant.

[15] A FIPS energy scan implicitly integrates over the instrument FOV to give an observed distribution as a function of measured speed, $f_{\text {obs }}(v)$. This integration can be explicitly written as

$$
f_{\text {obs }}(v)=\int_{0}^{\pi / 2} \sin \theta d \theta \int_{0}^{2 \pi} d \phi \cdot f(v, \theta, \phi) .
$$

[16] Equation (2) can be integrated analytically under the assumption of supersonic flow and a center of the distribution near the edge of the sunshade (see Appendix B). From this solution, the measured quantities $F_{0}, F_{1}$, and $F_{2}$ may be calculated and manipulated to produce the observed density $n_{\mathrm{obs}}<n_{\mathrm{o}}$ and estimates of $v_{\mathrm{o}}$ and $v_{\mathrm{th}}$ directly from FIPS measurements:

$$
\begin{aligned}
& F_{0}=\int_{\mathrm{o}}^{\infty} f_{\mathrm{obs}}(v) \cdot v^{2} d v \approx n_{\mathrm{obs}}, \\
& F_{1}=\left\langle\frac{1}{v}\right\rangle=\int_{\mathrm{o}}^{\infty} \frac{1}{v} \cdot f_{\mathrm{obs}} \cdot v^{2} d v \approx \frac{n_{\mathrm{obs}}}{v_{\mathrm{o}}}, \text { and } \\
& F_{2}=\left\langle\frac{\left(v-v_{\mathrm{o}}\right)^{2}}{v}\right\rangle=\int_{\mathrm{o}}^{\infty} \frac{\left(v-v_{\mathrm{o}}\right)^{2}}{v} \cdot f_{\mathrm{obs}} \cdot v^{2} d v \approx \frac{n_{\mathrm{obs}} \cdot v_{\mathrm{th}}^{2}}{v_{\mathrm{o}}} .
\end{aligned}
$$

$n_{\mathrm{obs}}, v_{\mathrm{o}}$, and $v_{\text {th }}$ can be recovered through manipulations of these moments,

$$
\begin{aligned}
n_{\mathrm{obs}} & =F_{0}, \\
v_{\mathrm{o}} & =\frac{F_{0}}{F_{1}}, \text { and } \\
v_{\mathrm{th}} & =\sqrt{\frac{F_{2}}{F_{1}}} .
\end{aligned}
$$

[17] These relations apply to any measured ion species, providing that the proper conversion from instrument energy to ion velocity is determined. Similar moments are used to derive solar wind parameters for observations from ACE/ SWICS and Ulysses/SWICS [von Steiger et al., 2000].

\subsubsection{Kappa Distributions}

[18] To first order, an isotropic Maxwell-Boltzmann distribution is a good description of the solar wind. However, a more general distribution function for solar wind plasmas is a kappa distribution [Vasyliunas, 1968],

$$
f_{\kappa}(v) \sim\left(1+\frac{1}{\kappa} \frac{\left(\mathbf{v}-\mathbf{v}_{\mathrm{o}}\right)^{2}}{2 v_{\mathrm{th}}^{2}}\right)^{-(\kappa+1)} .
$$

[19] The parameter $\kappa$, defined for $1.5<\kappa<\infty$, describes the shape of the velocity distribution away from a quasiMaxwellian core. A value $\kappa \approx 1.5$ gives a $v^{-5}$ power law tail, i.e., a strong suprathermal particle population commonly observed in the solar wind. This particular distribution will place an upper bound on that population, as the measured tail typically begins at several times the solar wind speed [Fisk and Gloeckler, 2006; Fisk et al., 2010].

[20] Higher $\kappa$ values describe distributions with weaker tails, and $\kappa \rightarrow \infty$ reduces the distribution to a tailless Maxwellian. However, for a low- $\kappa$ distribution, the results from Section 2.1.1 may not necessarily apply. The effect of these tails on the recovery of solar wind parameters will be to decrease velocity estimates and increase temperature estimates, due to an increase in measured events at angles far from the core that, in the instrument frame, are necessarily measured with a lower and wider energy range than for the core. Although indices can be created to characterize the strength of the suprathermal population, larger time accumulations for instrument measured events than those used in this work would be needed to obtain the required counting statistics for such an analysis. Therefore, as discussed below, valid recovery criteria are developed that are independent of any estimate of the size of the suprathermal population.

\subsubsection{Bi-Maxwellian Distributions}

[21] In addition to a suprathermal tail, solar wind distributions can exhibit structure with respect to the direction of the local magnetic field [Wolfe et al., 1966]. A simple model of these distributions is a bi-Maxwellian, with characteristic temperatures $T_{\|}$and $T_{\perp}$ (and corresponding $v_{\text {th, } \|}$ and $\left.v_{\mathrm{th}, \perp}\right)$ related to the overall plasma temperature by the relationship $T=1 / 3 \times\left(T_{\|}+2 T_{\perp}\right)$ [Hundhausen et al., 1970]. Here we assume that the magnetic field in the inner heliosphere is nearly radial to the Sun, such that the parallel 


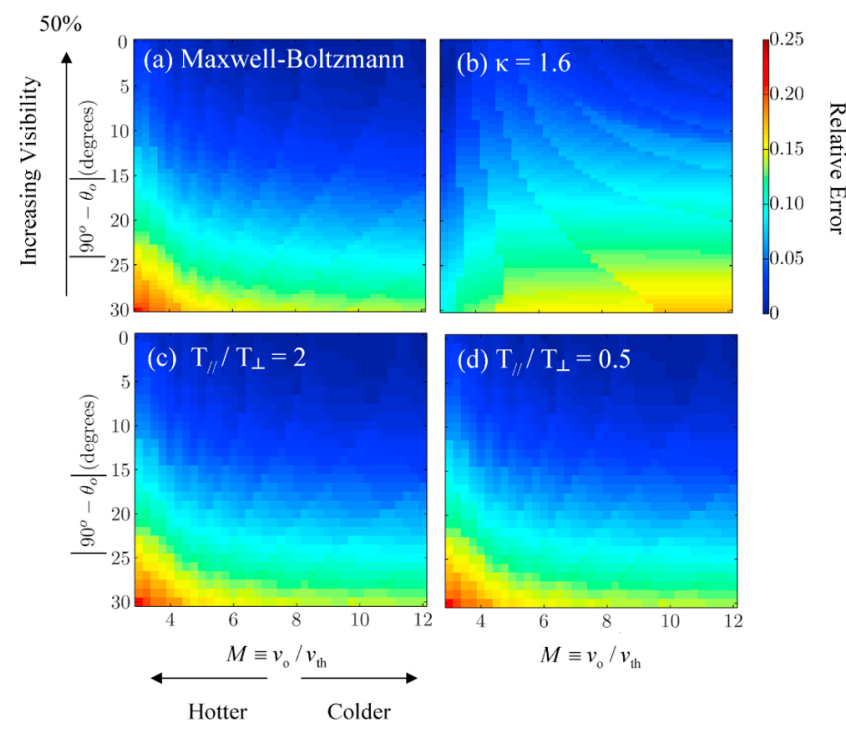

Figure 3. Relative error in recovered velocity for (a) isotropic Maxwell-Boltzmann, (b) kappa distribution with $\kappa=1.6$, (c) bi-Maxwellian with $T_{\|} / T_{\perp}=2$, and (d) bi-Maxwellian with $T_{\|} / T_{\perp}=0.5$. A decreasing $M \equiv v_{\mathrm{o}} / v_{\text {th }}$ indicates hotter, less supersonic plasma. $\left(90^{\circ}-\theta_{\mathrm{o}}\right)=0$ indicates that $50 \%$ of the distribution is visible, with a lower fraction visible at increasing angular distance from the core. For all distributions, when the core is within $20^{\circ}$ of the sunshade obstruction, the velocity is recovered to within $10 \%$ accuracy.

direction corresponds to the solar wind flow direction, giving a distribution of the form

$$
f(v) \sim \exp \left(-\frac{\left(v_{\|}-v_{\mathrm{o}}\right)^{2}}{2 v_{\mathrm{th}, \|}^{2}}-\frac{\left(v_{\perp}\right)^{2}}{2 v_{\mathrm{th}, \perp}^{2}}\right)
$$

where $v_{\|}$and $v_{\perp}$ are the components of the velocity in the directions parallel and perpendicular to the local magnetic field.

[22] Previous studies of inner heliospheric plasmas yield $T_{\|} / T_{\perp}$ in the range $10^{-1}$ to $10^{1}$ that can vary with different ion species [Marsch et al., 1982a, 1982b]. Although not modeled in detail here, a substantial heat flux component will add an asymmetry into the distribution, usually along the parallel direction [ $L i, 1999]$, resulting in a shift of the mean velocity and an enhanced thermal velocity due to a widening of the distribution. These heat flux contributions, like temperature anisotropy, often exhibit structure with respect to the magnetic field.

\subsection{Recovery Error Estimates}

[23] To quantify the recovery errors in $v_{\mathrm{o}}$ and $v_{\text {th }}$, data from synthetic distributions were analyzed with the methodology of the previous section. Four sets of distributions were generated: (1) an isotropic Maxwell-Boltzmann distribution, (2) a kappa distribution with $\kappa=1.6$, (3) a bi-Maxwellian with $T_{\|} / T_{\perp}=2$, and (4) a bi-Maxwellian with $T_{\|} / T_{\perp}=0.5$. Each set consists of distributions with varying values of $M \equiv$ $v_{\mathrm{o}} / v_{\text {th }}$ and $\theta_{\mathrm{o}}$ values. A value of $v_{\mathrm{o}}=450 \mathrm{~km} / \mathrm{s}$ was selected with $M$ varying from 3 to 12 and $\theta_{\mathrm{o}}$ varying from $90^{\circ}$ to $120^{\circ}$.
[24] For a given $M$ and $\theta_{\mathrm{o}}$, the distribution was sampled over $\theta=[0, \pi / 2]$ and $\phi=[0,2 \pi]$ at $1^{\circ}$ resolution and accumulated to form $f_{\text {obs }}(v)$. This $f_{\text {obs }}(v)$ was integrated following equation (2) to find $F_{0}, F_{1}, F_{2}$ and subsequently estimates for $v_{\mathrm{o} \text {,recovered }}$ and $v_{\mathrm{th} \text {,recovered }}$. These estimates were compared with the known input values of $v_{\mathrm{o}}$ and $v_{\mathrm{th}}$, and the relative error between them was calculated from

$$
E_{\mathrm{vo}}\left(M, \theta_{\mathrm{o}}\right)=\frac{\left|v_{\mathrm{o}}-v_{\mathrm{o}, \text { recovered }}\left(M, \theta_{\mathrm{o}}\right)\right|}{v_{\mathrm{o}}}
$$

and

$$
E_{\mathrm{vth}}\left(M, \theta_{\mathrm{o}}\right)=\frac{\left|v_{\mathrm{o}} / M-v_{\mathrm{th}, \text { recovered }}\left(M, \theta_{\mathrm{o}}\right)\right|}{v_{\mathrm{o}} / M} .
$$

[25] The recoveries tested here are applicable to multiple ion species in the same plasma. Although different species may have different bulk and/or thermal velocities, only motion of the distribution core with respect to the plane of the sunshade results in changes to $\theta_{\text {o }}$, i.e., only one dimension of ion motion can affect $\theta_{\mathrm{o}}$. Ion species with different solar wind speeds, for example, can still have the same $\theta_{\mathrm{o}}$ values, and consequently their errors are expected to scale with one another, allowing for detailed comparison between them. In addition, included in the conversion from measured events to phase space density is a $v^{4}$ dependent factor that, for lower density plasmas, can limit the recovered $f_{\text {obs }}(v)$ values at lower measured energies [von Steiger et al., 2000]. This effect was not included as part of this analysis because it is density dependent and a second order effect.

\subsubsection{Bulk Velocities}

[26] For almost all distributions visible to within $20^{\circ}$ of the core, regardless of the suprathermal population, the error in recovered velocity is less than $10 \%$, as demonstrated in Figure 3, which shows the relative error in recovered bulk velocities for all sets of analyzed distributions. Small-scale structure in these images is because multiple pairs of $M$ and $\theta_{\mathrm{o}}$ values produce similar distributions. Because of the finite sensitivity of FIPS and typical solar wind plasma densities in the inner heliosphere [Marsch et al., 1982b], it is unlikely that any distribution centered more than $20^{\circ}$ away from the sunshade obstruction will generate any noticeable measured events within the detector. It is therefore expected that, under the stated assumptions, FIPS can produce accurate estimates of solar wind speeds for any measured solar wind distribution.

\subsubsection{Thermal Velocities}

[27] As expected from Appendix A, and shown in Figure $4 \mathrm{a}$, the error in recovered thermal velocity is very small for the isotropic Maxwell-Boltzmann distribution and is relatively independent of the visible fraction of the distribution function. A strong suprathermal tail, however (Figure 4b), creates substantial errors in recovered thermal velocity except for either very hot $(M \approx 3)$ distributions or when almost half of the distribution function is visible. For the bi-Maxwellian cases, the recovered thermal velocity is dominated by the $v_{\mathrm{th}, \|}$ component, specifically for $T_{\|} / T_{\perp}>1$, since the integration is most sensitive to contributions from the flow/magnetic field direction. A substantial temperature anisotropy, therefore, can lead to a bias in recovered thermal 


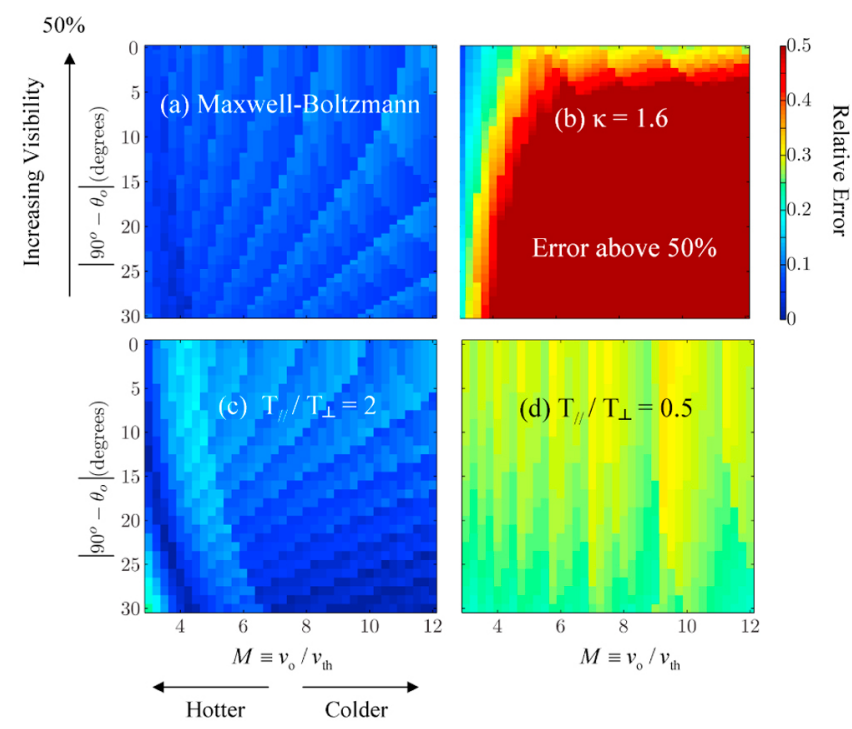

Figure 4. Relative error in recovered thermal velocities for (a) isotropic Maxwell-Boltzmann, (b) kappa distribution with $\kappa=1.6$, (c) bi-Maxwellian with $T_{\|} / T_{\perp}=2$, and (d) biMaxwellian with $T_{\|} / T_{\perp}=0.5$. For the Maxwell-Boltzmann case, the recovered thermal velocity is within $10 \%$ independent of the fraction of the distribution function that is visible to FIPS. For the $\kappa=1.6$ case, however, the strong suprathermal tail creates large changes in recovered $v_{\text {th }}$ with angular distance from the core. The recovered thermal velocities for the bi-Maxwellian cases are most sensitive to $v_{\mathrm{th}, \|}$, especially for $T_{\|} / T_{\perp}>1$.

velocity independent of the fraction of the distribution visible.

[28] Although the errors for the $\kappa=1.6$ case may be discouraging, they can nonetheless be used to aid in identification of periods of recoverable thermal velocities. Consider the turbulent solar wind moving in and out of the FIPS FOV. As this movement occurs, the instantaneous $\theta_{\mathrm{o}}$ value changes. Following the results in Figure 4b, such movement in the presence of a strong suprathermal population will create sharp changes in the recovered $v_{\text {th }}$ value as a function of time. Therefore, despite a lack of knowledge of the precise values of $\theta_{\mathrm{o}}$ or $\kappa$, if the recovery of thermal velocity is restricted to time periods where there is a stably recovered $v_{\text {th }}$ with time, it is likely that either FIPS is observing a nearMaxwellian distribution or that it is close to the solar wind core such that the effects of the suprathermal particle population will not affect the recovery. During these time periods, and with the given assumptions, it is estimated that the recovery of thermal velocity will be accurate to within $10 \%$.

\subsection{Recovery Criteria}

[29] From Section 2.2, bulk and thermal velocities of solar wind ions can be recovered to within $10 \%$ accuracy. However, the solar wind is not the only plasma population measured by FIPS as MESSENGER orbits Mercury, passing through the planet's magnetosphere twice per day during the primary orbital mission. Not only are criteria needed to determine whether or not we can expect accurately recovered solar wind parameters for a particular ion species, but the plasma population that is being measured by the instrument must be determined.

[30] The highest count rates in the instrument correspond to proton events. Therefore, a set of filters that examine the plasma properties of the measured protons were used to determine whether the solar wind distribution was being observed. Less abundant species such as $\mathrm{He}^{2+}$ and other heavy ions were recovered only during these times. $\mathrm{H}^{+}$ velocities were recovered for each instrument energy scan, averaged over approximately $1 \mathrm{~min}$ or $10 \mathrm{~s}$ depending on instrument operating mode. Longer time-averaged velocities of $\mathrm{H}^{+}$are averages of these individual scans. Heavy ion measurements, due to limited statistics, were summed over several scans before recovering their velocities. The thresholds below which velocities were not recovered due to insufficient statistics were selected as $n_{\mathrm{obs}}<5 \times 10^{-3} \mathrm{~cm}^{-3}$ for bulk velocity and $n_{\mathrm{obs}}<1.5 \times 10^{-2} \mathrm{~cm}^{-3}$ for thermal velocities.

[31] Because of the limitations of mass resolution for FIPS, heavy ion charge states such as $\mathrm{O}^{6+}$ and $\mathrm{C}^{5+}$ cannot be easily separated. Consequently, events from all ions with $\mathrm{m} / \mathrm{q}$ between $\sim 2$ and $3 \mathrm{amu} / \mathrm{e}$ were accumulated together. These events, however, will typically be dominated by $\mathrm{O}^{6+}$ and $\mathrm{C}^{5+}$, the most abundant heavy ion species in the solar wind [von Steiger et al., 2000]. For this reason, the average heavy ions will be denoted as $\langle O C\rangle$. Since the conversion from energy-per-charge to velocity depends on $m / q$, an average solar wind $m / q$ of 2.5 was assumed on the basis of abundances reported by von Steiger et al. [2000]. This factor may introduce additional errors on the order of about $5 \%$ to the determination of the average heavy ion velocities.

[32] Bulk velocities were recovered from FIPS measurements if: (1) sufficient numbers of $\mathrm{H}+$ events were measured to obtain $10 \%$ peak widths, (2) MESSENGER was outside of Mercury's bow shock as determined by data from magnetic field measurements (R. M. Winslow et al., Mercury's magnetopause and bow shock from MESSENGER observations, submitted to Journal of Geophysical Research, 2012), (3) the measured $\mathrm{H}^{+}$energy distribution was singly peaked, (4) the mean incident location of events was calculated to be within $30^{\circ}$ of the radial direction, and (5) more than two consecutive energy scans met criteria (1-4). Thermal velocities were recovered if criteria (1-5) were met with the added restriction that there must be recoverable thermal velocities from at least five consecutive energy scans and that the standard deviation of those velocities stayed below 5 $\mathrm{km} / \mathrm{s}$, indicating a stable recovery. Heavy ion parameters were recovered when the above criteria were met for $\mathrm{H}^{+}$and when sufficient numbers of heavy ion events were measured.

[33] As an example, Figure 5 shows a period of recovered bulk and thermal velocities for $\mathrm{H}^{+}, \mathrm{He}^{2+}$, and $\langle O C\rangle$ for the period from 21 June to 1 July 2011. The stream structure of the solar wind [McComas et al., 1998b] as well as plasma temperatures consistent with previous observations of inner heliospheric solar wind from Helios 1 and 2 [Freeman, 1988; Marsch et al., 1982b] were recovered from the filtered dataset. The solar wind parameters derived here were also compared with those generated by the Wang-SheeleyArge ENLIL model (D. N. Baker et al., Solar wind forcing at Mercury: WSA-ENLIL model results, submitted to 

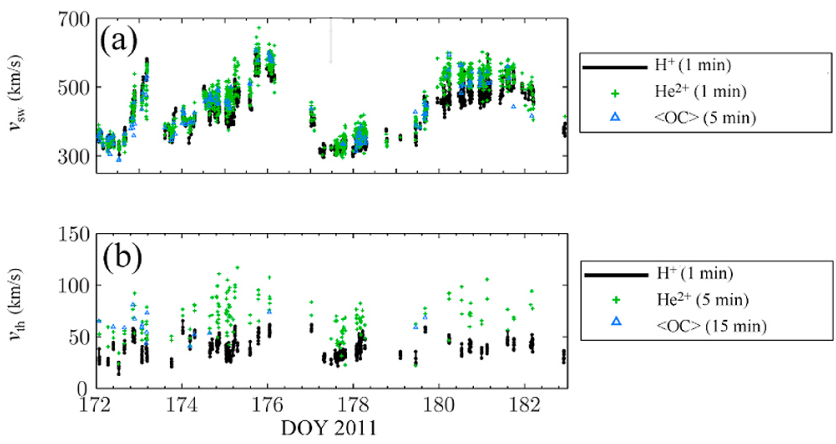

Figure 5. Recovered (a) bulk velocities and (b) thermal velocities of $\mathrm{H}^{+}, \mathrm{He}^{2+}$, and $\langle O C\rangle$ for the period from 21 June to 1 July 2011. These filtered datasets show typical solar wind structure, with speeds and temperatures characteristic of inner heliospheric plasma. Time accumulations for each data product are indicated.

Journal of Geophysical Research, 2012), and they were found to be in good agreement during quiet solar conditions.

\section{Heavy Ion Measurements in the Inner Heliosphere}

[34] With bulk and thermal velocities recoverable for both solar wind protons and heavy ion species, the effects of propagating Alfvénic fluctuations and average heavy ion kinetic properties can be characterized in the inner heliosphere (solar distance $R<0.5 \mathrm{AU}$ ) with data from both the FIPS and the Magnetometer (MAG) [Anderson et al., 2007] on MESSENGER.

\subsection{Identification of Alfvénic Turbulence}

[35] Alfvénic fluctuations are ubiquitous structures in the solar wind [Coleman, 1968; Belcher and Davis, 1971], particularly in fast streams associated with coronal holes [Smith et al., 1995]. These fluctuations have been observed in situ as close as $R=0.3$ AU by Helios [Grappin et al., 1990]. They are identified as periods of nearly constant magnetic field magnitude $|\mathbf{B}|$ and plasma density $n$ (mass density $\rho$ ), with correlations between changes in the measured solar wind velocity and magnetic field vector, such that

$$
\delta \mathbf{v} \equiv \mathbf{v}-\langle\mathbf{v}\rangle
$$

and

$$
\delta \mathbf{B} \equiv \mathbf{B}-\langle\mathbf{B}\rangle .
$$

with $\delta \mathbf{v}$ proportional to $\delta \mathbf{B}$, where $\mathbf{v}$ is the solar wind velocity vector and $\mathbf{B}$ is the magnetic field vector. The proportionality constant $(\delta v / \delta B)$ is related to the wave velocity $\mathbf{v}_{\mathrm{w}}$ of the propagating fluctuation by the relation $[B$. E. Goldstein et al., 1995]

$$
\mathbf{v}_{\mathrm{w}}=\frac{\delta v}{\delta B} \mathbf{B}
$$

[36] For an ideal Alfvén wave, $\delta \mathbf{v}= \pm \delta \mathbf{B} / \sqrt{4 \pi \rho}$, leading to a wave propagating with the Alfvén speed along the direction of $\mathbf{B}$. Because of the large uncertainties in $\rho$ from FIPS measurements, the local Alfvén speed cannot be reliably determined. However, from the correlation between $\delta \mathbf{v}$ and $\delta \mathbf{B}$, the effective wave speed from (11) is readily calculable.

[37] For large-amplitude nonlinear Alfvénic fluctuations, correlations in $\mathbf{v}$ and $\mathbf{B}$ are present in all vector components, not just those transverse to the direction of propagation [Belcher and Davis, 1971]. However, any analysis of FIPS observations is restricted to the direction radial to the Sun, as the recovered speed is approximately equal to the radial component of the solar wind velocity. Therefore, Alfvénic fluctuation events will be identified as time periods during which the measured $v_{\mathrm{H}^{+}}$correlates with the radial (with respect to the Sun) component of the magnetic field, $B_{\mathrm{R}}$, and the magnetic field magnitude $|\mathbf{B}|$ is nearly constant. The constant of proportionality between $\delta v_{\mathrm{H}^{+}}$and $\delta B_{\mathrm{R}}$ has been used to derive the radial propagation speed of the fluctuation from equation (11).

\subsection{Differential Streaming of Heavy Ions}

[38] Two periods of propagating Alfvénic fluctuations identified in the FIPS dataset on 19 April 2011, from 00:00:00 to 02:40:00 UTC, and 28 August 2011, from 14:00:00 to 15:00:00 UTC, are shown in Figures 6 and 7, respectively, with proton and heavy ion velocities from FIPS and corresponding magnetic field vector measurements from MAG. In each case, there is a nearly constant $|\mathbf{B}|$, consistent with incompressible turbulence. There are also observable correlations between $v_{\mathrm{H}^{+}}$and $B_{\mathrm{R}}$ with correlation coefficients $r^{2}=0.62$ and 0.73 for the 19 April 2011 and 28 August 2011 events, respectively. The time resolution of the data from each event varies because of different FIPS instrument operating modes. For each case, the maximum available time

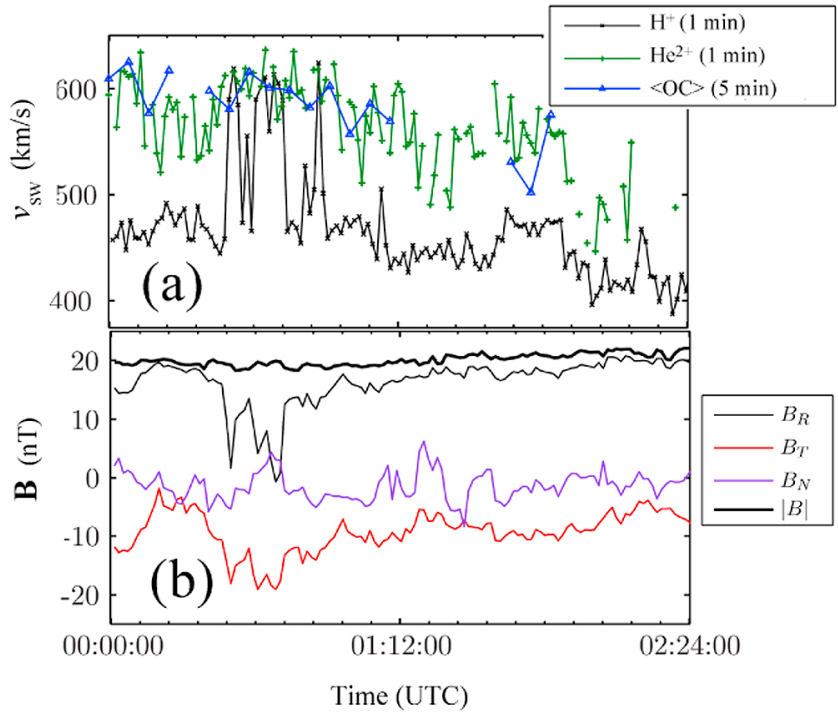

Figure 6. Alfvénic fluctuation event for 19 April 2011, from 00:00:00 to 02:40:00 UTC, in instrument survey mode. (a) FIPS-measured bulk speeds of $\mathrm{H}^{+}, \mathrm{He}^{2+}$, and $\langle O C\rangle$ show differential streaming of heavy ions with respect to solar wind protons. Time accumulations for each data product are indicated. (b) Magnetic field measurements from MAG (1-min averages) indicate strong correlations of $B_{\mathrm{R}}$ and $v_{\mathrm{H}^{+}}$ with a nearly constant $|\mathbf{B}|$. 


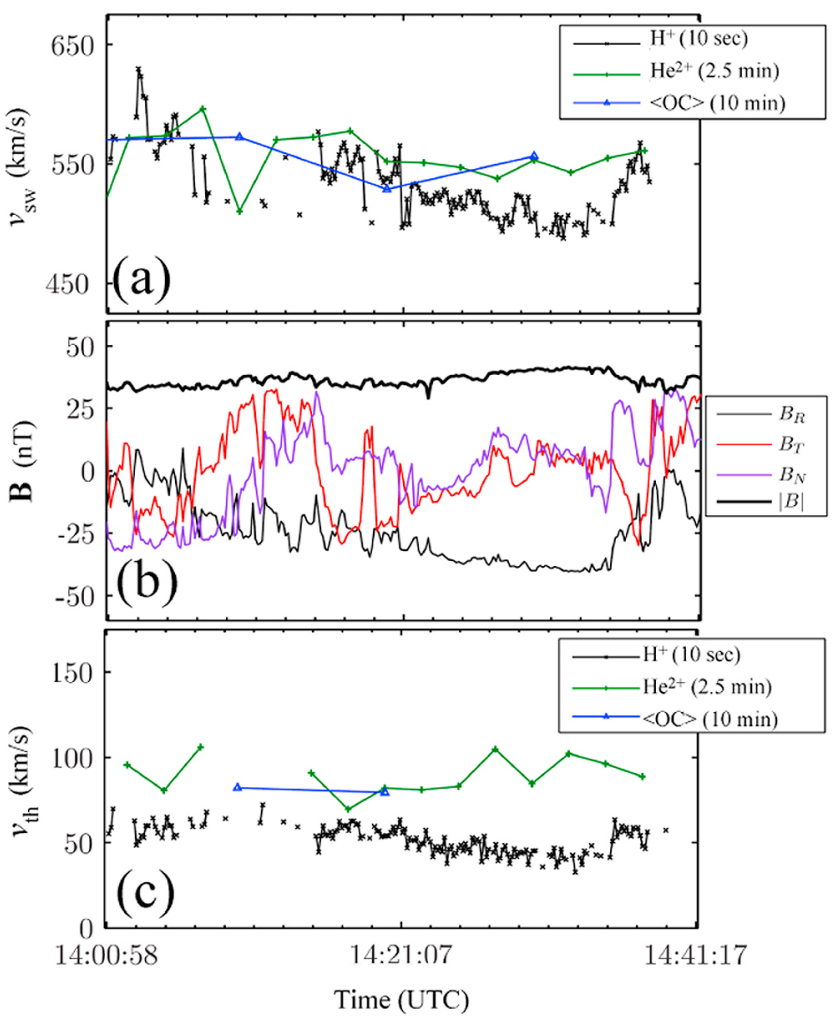

Figure 7. ( $\mathrm{a}$ and $\mathrm{b}$ ) Same as Figures $6 \mathrm{a}$ and $6 \mathrm{~b}$ but for 28 August 2011, from 14:00:00 to 15:00:00 UTC. (c) Corresponding thermal velocities. During this time period, FIPS was in its "burst" scanning mode. Time accumulations for each data product are indicated. The magnetic field data were averaged over 10 -s intervals in order to observe the fine structure of the $B_{\mathrm{R}}-v_{\mathrm{H}^{+}}$correlation.

resolution was used to derive $v_{\mathrm{H}^{+}}$in order to resolve the fine structure of the fluctuations. The thermal velocities for the 19 April event did not meet the stability criteria for a reliable recovery and are, as a consequence, not included in this analysis. However, for the 28 August event, the $\mathrm{H}^{+}, \mathrm{He}^{2+}$, and $\langle O C\rangle$ thermal velocities are available and are shown in Figure 7.

[39] From previous observations [von Steiger and Zurbuchen, 2006], it is expected that the timescale of interactions between solar wind ions and propagating waves scales as $(q / m)^{\alpha}$, where $\alpha$ is related to the spectral slope of the magnetic field fluctuations. Heavier ions should therefore scatter quickly into the frame of the waves or of the Alfvénic turbulence, leading to a motion relative to protons, and also excessive heating. In addition, these ions are heated such that their thermal velocities approach that of the wave speed. This process results in differential streaming of heavy ions with respect to the protons in the direction of the magnetic field [Asbridge et al., 1976; Marsch et al., 1982a; Zurbuchen et al., 2000; Berger et al., 2011]. Since waves tend to propagate outward from the Sun [Roberts et al., 1987; Gosling et al., 2009], such streaming results in an increased measured solar wind speed of heavy ions.

[40] This wave-particle scattering is incomplete, however, and particles have been observed to stream with only a fraction of the propagating wave speed. Some weak correlation between the $\mathrm{He}^{2+}$ speed and $B_{\mathrm{R}}$ in Figures 6 and 7 is further evidence of this incomplete scattering process, as only particles not traveling in the frame of the wave could be susceptible to such interactions. The constant of proportionalities between $\delta v_{\mathrm{H}^{+}}$and $\delta B_{\mathrm{R}}$ for the events in Figures 6 and 7 are $-9.2 \mathrm{~km} / \mathrm{s} \cdot \mathrm{nT}$ and $-2 \mathrm{~km} / \mathrm{s} \cdot \mathrm{nT}$, respectively, leading to maximum calculated wave speeds of $175 \mathrm{~km} / \mathrm{s}$ and $80 \mathrm{~km} / \mathrm{s}$. The $\mathrm{He}^{2+}$ bulk radial velocities appear to stream ahead of the protons at a fraction of this speed, approximately $70 \%$, for both the 19 April and 28 August events, consistent with the results of Marsch et al. [1982a]. The $\langle O C\rangle$ bulk velocities also appear to lead the protons by similar factors, though with the longer required accumulation time and consequently fewer available data points for heavy ions, this relationship is less clear.

[41] For the 28 August event, the heavy ion thermal velocities also lead that of the proton velocity, indicating substantial heating for each species when compared with $\mathrm{H}^{+}$. The average thermal velocity of heavy ions during the event is approximately $90 \mathrm{~km} / \mathrm{s}$, consistent with particles scattered into the frame of the propagating wave. Ions with markedly different thermal speeds could result in biases in their recovered bulk velocities, following the derivation in Appendix A. However, the effect of this bias would be to decrease the recovered speed of the heavy ions, such that their relative streaming speed would be slightly underestimated.

[42] Although the full time interval of each event appears to have a strong $v_{\mathrm{H}^{+}}-B_{\mathrm{R}}$ correlation, there are short periods within each period with no observed differential streaming, i.e., $v_{\mathrm{H}^{+}}=v_{\text {heavy. }}$. These periods can be understood as marking changes in the propagation direction of the fluctuations. Nominally, Alfvén waves are transverse perturbations that travel parallel to the mean field. However, for nonlinear high-amplitude waves, the propagation direction may be altered by large fluctuations. During these events, although the wave speed $\left|\mathbf{v}_{\mathrm{w}}\right|$ must stay constant, the individual components can change to accommodate an evolving direction of propagation. The periods in Figures 6 and 7 at which $v_{\mathrm{H}^{+}}=v_{\text {heavy }}$ correspond to times when the magnetic field became notably nonradial, i.e., $\left|B_{\mathrm{T}}\right|,\left|B_{\mathrm{N}}\right|>\left|B_{\mathrm{R}}\right|$, in agreement with the analysis of Berger et al. [2011] at 1 AU. With the direction of propagation no longer in the radial direction, the differential speed of heavy ions and $\mathrm{H}^{+}$measured by FIPS should be small. These observations indicate that Alfvénic fluctuations travel along the local magnetic field direction. High time-cadence measurements are vital for resolving this phenomenon, as average heavy ion velocities over the timescale of the fluctuations can wash out this effect.

\subsection{Average Heavy Ion Kinetic Properties}

[43] Because of the limited solar wind dataset of FIPS, it is not possible to identify all potential Alfvénic fluctuation events from instrument observations. Consequently, wave speeds can be identified only for a handful of events for which there are large segments of continuous measurements. Nonetheless, it is possible to investigate average trends of heavy ion dynamics. Such data for the inner heliosphere $(R<0.5$ AU) from FIPS can be compared with previous observations at $1 \mathrm{AU}$. For the full time range available in 2011, average bulk and thermal velocities were recovered from FIPS measurements. 

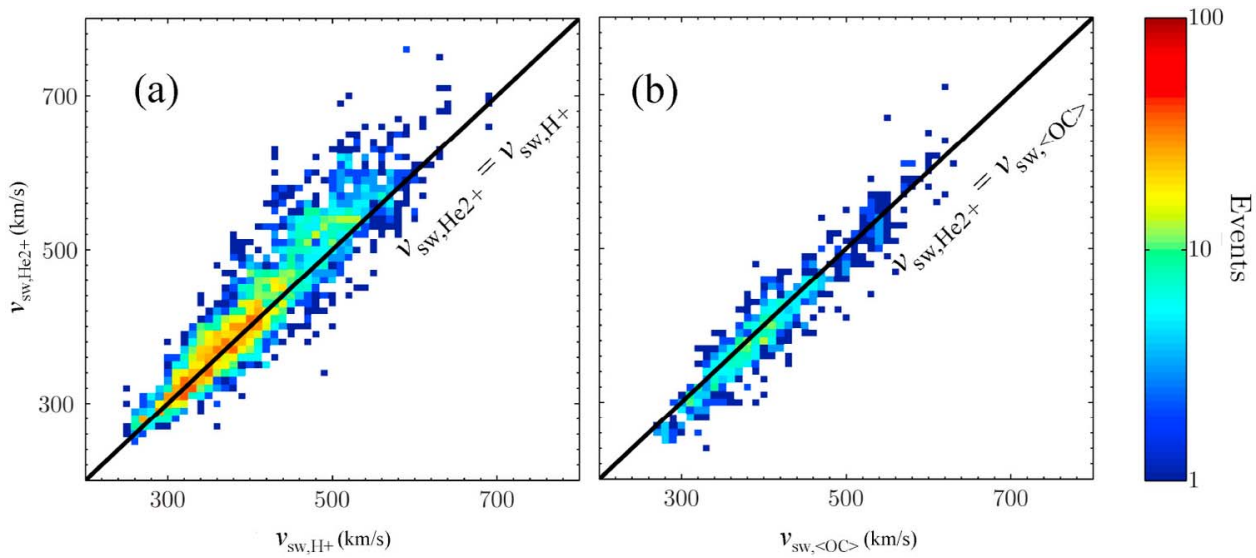

Figure 8. $v_{\mathrm{He}^{2+}}$ as a function of (a) $\mathrm{H}^{+}$bulk velocities and (b) $\langle O C\rangle$ bulk velocities measured by FIPS. For lower solar wind speeds $(v<450 \mathrm{~km} / \mathrm{s})$, helium and hydrogen travel together. For higher speed winds $(v>450 \mathrm{~km} / \mathrm{s})$, at which Alfvénic turbulence becomes more prevalent, the helium ions stream ahead of the protons. Helium and $\langle O C\rangle$ flow together for all measured solar wind speeds.

\subsubsection{Bulk Flow}

[44] The average bulk velocity of $\mathrm{He}^{2+}$ compared with those of $\mathrm{H}^{+}$and $\langle O C\rangle$ are shown in Figure 8. At lower solar wind speeds $(v<450 \mathrm{~km} / \mathrm{s}), v_{\mathrm{H}^{+}} \approx v_{\mathrm{He}^{2+}}$, indicating the absence of substantial differential streaming. However, for higher speeds $(v>450 \mathrm{~km} / \mathrm{s})$, the helium ions consistently stream ahead of the protons, a trend in good agreement with increased Alfvénic wave activity in coronal-hole winds. For all measured solar wind speeds, however, there does not appear to be any average streaming between alpha particles $(m=4 \mathrm{amu})$ and heavier ions ( $m=12-16 \mathrm{amu})$. Such behavior is consistent with an $m / q$-dependent physical processes, in which higher- $m / q$ ions all flow with the protons in the slower solar wind and all stream ahead of the protons with similar velocities during times of increased Alfvénic wave activity in the fast wind.

\subsubsection{Thermal Properties}

[45] The thermal velocities are compared between $\mathrm{He}^{2+}$ and $\mathrm{H}^{+}$and between $\mathrm{He}^{2+}$ and $\langle O C\rangle$ in Figure 9. Lines indicating thermal equilibrium, equal thermal speeds, and the maximum differential thermal speeds $\left(v_{\mathrm{th}, O^{5+}} / v_{\mathrm{th}, \mathrm{H}} \approx 2.33\right.$ at 3.5 solar radii [Kohl et al., 1998] observed close to the Sun by UVCS are included in the figure. Although UVCS observed temperatures only of neutral hydrogen, similar trends are expected to hold for that of $\mathrm{H}^{+}$. Here it is also assumed that the observations of $\mathrm{O}^{5+}$ are representative of coronal $\mathrm{He}^{2+}$, since no corresponding spectroscopic data are available. Heavy ions seen by MESSENGER are observed to be substantially hotter than protons, with few observed alpha particles in thermal equilibrium with the protons, in contrast to analogous observations at 1 AU. The maximum $v_{\text {th, } \mathrm{He}^{2+}} / v_{\text {th, } \mathrm{H}^{+}}$from FIPS, however, stayed within the bounds of UVCS observations, indicating that a substantial additional heating source outside the solar wind acceleration region may not be required to explain FIPS observations. Further, Figure $9 \mathrm{~b}$ indicates that the thermal velocities for $\mathrm{He}^{2+}$ and $\langle O C\rangle$, like their bulk velocities, are nearly identical.

[46] For Parker spiral geometry and a solar wind density that scales as $1 / R^{2}$, the Alfvén speed $\left(v_{\mathrm{A}}\right)$ increases by about a factor of 2 from 1 AU to $0.4 \mathrm{AU}$, the average distance of MESSENGER from the Sun during the time period of this
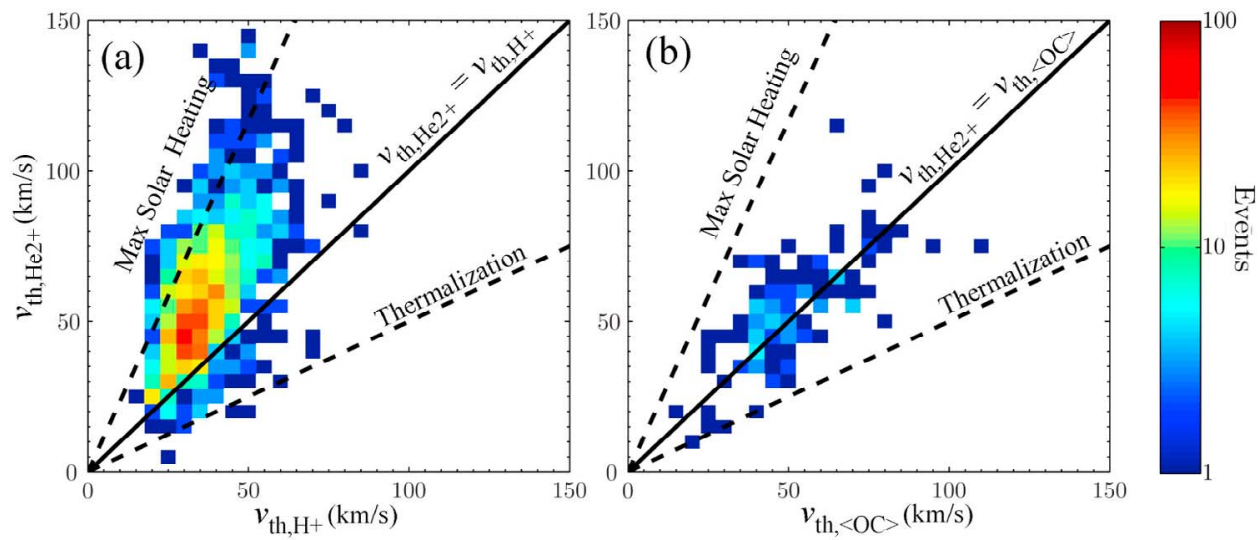

Figure 9. $v_{\mathrm{th}, \mathrm{He}^{2+}}$ as a function of (a) $\mathrm{H}^{+}$thermal velocities and (b) $\langle O C\rangle$ thermal velocities measured by FIPS. Helium is substantially heated with respect to the protons; on average $T_{\mathrm{He}^{2+}} / T_{\mathrm{H}^{+}} \approx 9$. Both $\mathrm{He}^{2+}$ and $\langle O C\rangle$ have similar thermal speeds, indicating substantial heating of the heavier ions as well. 


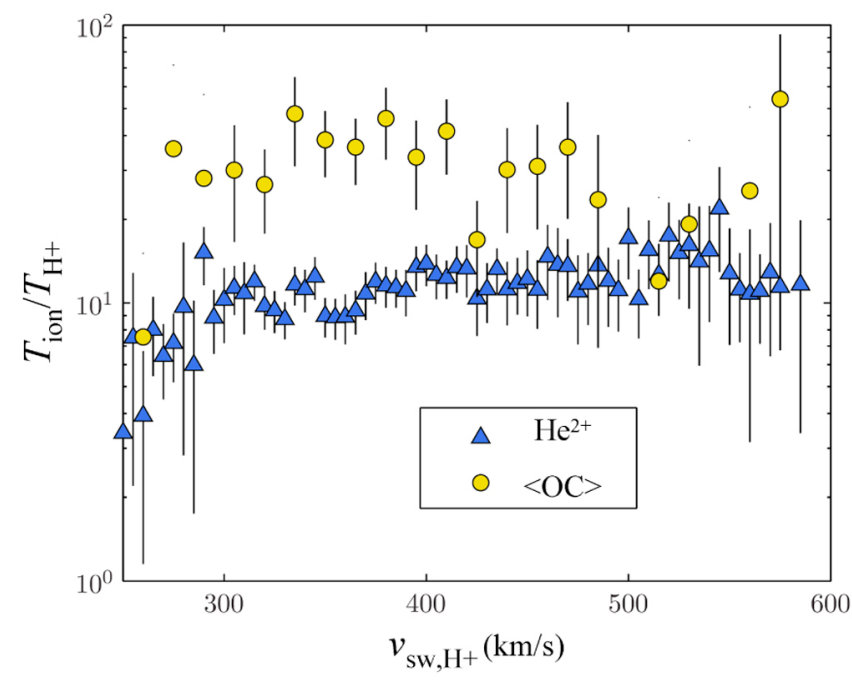

Figure 10. $T_{\mathrm{ion}} / T_{\mathrm{H}^{+}}$as a function of solar wind speed for $\mathrm{He}^{2+}$ and $\langle O C\rangle$. The error bars for each point indicate counting uncertainty, with the percent error scaling as the inverse square root of the number of measurements, $1 / \sqrt{N}$. With decreasing solar wind speed, i.e., less Alfvénic turbulence, the plasma tends toward a lower temperature ratio. The temperature ratio $T_{\langle\mathrm{OC}\rangle} / T_{\mathrm{He}^{2+}}$ is approximately equal to $m_{\langle\mathrm{OC}\rangle} /$ $m_{\mathrm{He}^{2+}}$, implying similar thermal speeds for heavy ions, as shown in Figure 9b.

study. The proton thermal speed, however, for a measured $R^{-0.9}$ scaling for temperature in the fast solar wind in the inner heliosphere [Totten et al., 1995], increases only by a factor of approximately 1.5 from $1 \mathrm{AU}$ to $0.4 \mathrm{AU}$, such that the overall ratio $v_{\mathrm{A}} / v_{\mathrm{th}, \mathrm{H}^{+}}$will increase by a factor of 1.3 , i.e., the proton thermal velocity does not scale with the Alfvén speed. As discussed in Section 3.2, $\mathrm{He}^{2+}$ ions can be heated by propagating Alfvénic fluctuations such that their thermal speed approaches that of the Alfvén speed. It follows that the helium thermal velocity during these times should scale with the Alfvén velocity. At $1 \mathrm{AU}, T_{\mathrm{He}^{2+}} / T_{\mathrm{H}^{+}}=4$, i.e., equal proton and alpha thermal speeds correspond to time periods of increased magnetic wave activity and differential streaming [Kasper et al., 2008]. Inside $1 \mathrm{AU}$, therefore, if $\mathrm{He}^{2+}$ particles have their thermal speeds ordered by the Alfvén speed, a peak in the ratio $T_{\mathrm{He}^{2}+} / T_{\mathrm{H}^{+}} \approx 8$ is expected. From Helios, $T_{\mathrm{He}^{2+}} / T_{\mathrm{H}^{+}}$in the inner heliosphere was 5-6 [Marsch et al., 1982a]. The average $T_{\mathrm{He}^{2+}} / T_{\mathrm{H}^{+}}$from FIPS observations, $\sim 11$, is higher than this scaled ratio and nearly twice that of the previous Helios observations.

[47] Temperature enhancements measured by FIPS as a function of $v_{\mathrm{H}^{+}}$for $\mathrm{He}^{2+}$ and $\langle O C\rangle$ are shown in Figure 10 for an average $\langle O C\rangle$ mass of $14.4 \mathrm{amu}$ [von Steiger et al., 2000]. In the fast wind, where Alfvénic turbulence is most prevalent, the temperature ratio between the heavy ions and protons is largest, with the maximum ratio approaching the maximum observed heavy ion heating from UVCS measurements [Kohl et al., 1998]. The minimum ratios occur for the lowest measured solar wind speeds, at which Coulomb collisions are expected to play an increasingly important role in heavy ion kinematics. The temperature ratio $T_{\langle O C\rangle} / T_{\mathrm{He}^{2+}}$ is approximately equal to $m_{\langle O C\rangle} / m_{\mathrm{He}^{2+}}$, consistent with results shown in Figure 9b, indicating that heavy ions move with similar thermal velocities. From this analysis, measurements of $T_{\text {ion }} / T_{\mathrm{H}^{+}}$in the inner heliosphere appear to be ordered by the solar wind regime, consistent with similar analysis at 1 AU by Kasper et al. [2008], but with substantially higher observed alpha particle temperatures.

[48] As discussed in Appendix A, the recovery of the thermal velocities of ion species relies on the assumption of an isotropic plasma. Although there may be biases in the recovered of thermal velocities from partially observed solar wind plasmas, any bias in the $T_{\mathrm{He}^{2+}} / T_{\mathrm{H}^{+}}$ratio must be due to a difference in biases between the alpha particles and the proton population. If substantial anisotropy is present in the $\mathrm{He}^{2+}$ particles with respect to those of $\mathrm{H}^{+}$, i.e., $T_{\mathrm{He}^{2+}, \|} / T_{\mathrm{H}^{+}, \|} \gg 1$, the ratio of the recovered thermal velocities could be biased. Furthermore, there may also be a larger or more variable suprathermal population for heavier ions than for $\mathrm{H}^{+}$, enhancing the estimates of alpha particle temperatures. Finally, the solar wind populations most easily measured by FIPS have higher plasma temperatures in general due to the tendency of colder distribution functions to remain hidden behind the spacecraft sunshade.

[49] As indicated by data gaps in the observations of Marsch et al. [1982a] close to the Sun, however, available Helios observations may exhibit an opposite bias. Alpha particles and protons become increasingly more difficult to separate with increasing $T_{\mathrm{He}^{2+}} / T_{\mathrm{H}^{+}}$ratios using only electrostatic deflection, leading to a possible explanation for the increased temperatures measured by MESSENGER when compared with Helios observations. Notwithstanding any bias, however, from FIPS observations, there are few recovered thermal velocities indicating that $\mathrm{He}^{2+}$ and $\mathrm{H}^{+}$are in thermal equilibrium, in striking contrast to data collected at $1 \mathrm{AU}$.

\subsubsection{Magnetic Turbulent Heating}

[50] To interpret the non-thermal heating and differential acceleration of heavy ions as a function of heliocentric distance, a comparison of two representative spectra of locally measured magnetic field data in the fast solar wind $\left(v_{\mathrm{H}^{+}} \approx\right.$ $500 \mathrm{~km} / \mathrm{s}$ ) at 0.37 and $1 \mathrm{AU}$ is shown in Figure 11. The ACE data were measured by the magnetometer on ACE [Smith et al., 1998] with a maximum time-resolution of 3 vectors/s. MESSENGER data were measured with a maximum timeresolution of 20 vectors/s. Spectral densities for the trace of the total power of fluctuations in the magnetic field components $\left(P_{\mathrm{Tr}}\right)$ and the total power of fluctuations in the magnetic field magnitude $\left(P_{|\mathbf{B}|}\right)$ are shown. For each spectrum, there is a power law fit in the inertial frequency range $10^{-2} \mathrm{~Hz}<f<$ $10^{-1} \mathrm{~Hz}$. Figure 11 also marks the gyrofrequencies of $\mathrm{H}^{+}$, $\mathrm{He}^{2+}$, and $\langle O C\rangle$.

[51] In the low-frequency inertial regime, the spectral shape of $P_{\mathrm{Tr}}$ is almost independent of heliocentric distance, consistent with Helios observations [Marsch, 1991]. The power law fits in this region show a slope consistent with Kolmogorov scaling, i.e., $f^{-5 / 3}$ for both spectra. However, the spectral power at $0.37 \mathrm{AU}$ is elevated by a factor of 20 in the frequency range $10^{-3} \mathrm{~Hz}<f<10 \mathrm{~Hz}$. Following the Wentzel-Kramers-Brillouin (WKB) approximation and under steady plasma conditions, $P_{|\mathbf{B}|}$ is expected to increase following a $R^{-3}$ dependence in the inner heliosphere [Isenberg and Hollweg, 1982], consistent with the observed change in $P_{|\mathbf{B}|}$. 

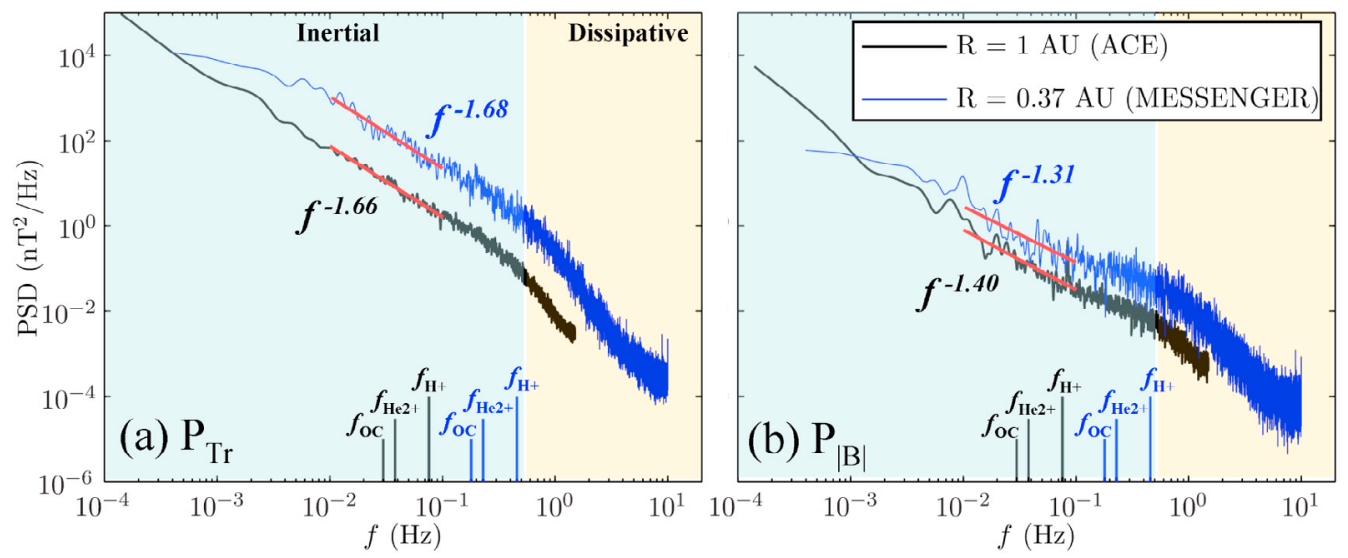

Figure 11. Representative high-resolution spectra for MESSENGER (in blue) and ACE (in black) during a time of coronal-hole-associated wind (ACE: 6 March 2011, 04:00:00-07:59:59 UTC; MESSENGER: 29 June 2011, 01:26:00-02:08:00 UTC). Power spectra are shown for (a) the trace of three-dimensional spectral density distributions of $\mathbf{B}$, i.e. $P_{\mathrm{Tr}}$ and (b) fluctuations in the magnitude of $\mathbf{B}$, i.e., $P_{|\mathbf{B}|}$. The local gyrofrequencies of key ions $\left(\mathrm{H}^{+}, \mathrm{He}^{2+}\right.$, and $\left.\langle O C\rangle\right)$ are computed from average $|\mathbf{B}|$ values during this time period, $\sim 5 \mathrm{nT}$ and $\sim 30 \mathrm{nT}$ for ACE and MESSENGER, respectively.

[52] As discussed in Section 3.1, one view of the fluctuations in $\mathbf{B}$ can be expressed as a combination of inward- and outward-propagating Alfvén waves that interact in a nonlinear fashion [Marsch, 1991; M. L. Goldstein et al., 1995]. Interactions between these large-amplitude waves and particles are expected to be the physical reason for the differential streaming of solar wind heavy ions. The spectral density of $P_{|\mathbf{B}|}$ is smaller than $P_{\mathrm{Tr}}$ by over two orders of magnitude, indicating that these inertial range fluctuations are approximately incompressible. This incompressibility is consistent with the observed properties of Alfvénic fluctuations for which $|\mathbf{B}|$ remains nearly constant but with large fluctuations in all individual components. However, the spectral slope of $P_{|\mathbf{B}|}$ is substantially flatter than that of $P_{\mathrm{Tr}}$, indicating increasingly compressible turbulence toward the transition to the dissipative range.

[53] At both ACE and MESSENGER, the transition from the incompressible inertial range to the compressible dissipation range manifests as a change in spectral slope near $f=0.5 \mathrm{~Hz}$. Although the heavy ion gyrofrequencies at $1 \mathrm{AU}$ are well within the inertial range, the corresponding gyrofrequencies at $R=0.37 \mathrm{AU}$ occur near this point. Despite important Doppler shifts not having been included in the labeling of ion gyrofrequencies in Figure 11, a few important conclusions can be drawn from this analysis: (1) the transition from inertial to dissipation ranges and the kinetic timescale of heavy ions and protons in the inner heliosphere evolve differently with heliocentric distance but span a similar band of frequencies; (2) solar wind heavy ions will necessarily interact with solar wind turbulence at lower frequencies, and consequently will always experience a substantially larger power level than that of the protons, e.g., $P_{\mathrm{Tr}}\left(f_{O C}\right) \approx 5 P_{\mathrm{Tr}}\left(f_{\mathrm{H}^{+}}\right)$in the inertial range; (3) there appears to be a distinct increase in the compressive nature of fluctuations in the frequency range near the ion gyrofrequencies in the inner heliosphere; and (4) for local heavy ion resonant interaction, one would expect spectral breaks at the locations of heavy ion gyrofrequencies. The absence of these markers suggests that either the majority of the heavy ion waveheating process occurs close to the Sun, or that the turbulent cascade masks any signature of a gradual heliospheric process.

\section{Concluding Remarks}

[54] Observations by the FIPS sensor on MESSENGER constitute the first measurements of the properties of heavier ions in the solar wind at $\sim 0.3 \mathrm{AU}$, and the first measurements of any solar wind properties at this heliocentric distance since observations by the Helios spacecraft 30 years ago. Our results, as in the case of the solar wind at $1 \mathrm{AU}$, are divided by the dominant source of turbulence, i.e., by fast solar wind, which has substantial Alfvénic turbulence, versus slow solar wind, which has more compressive turbulence. In the case of the slow wind, the protons and heavier ions tend to have the same flow speed and approximately equal temperatures, which were presumably set in collisiondominated processes in the solar corona. In the case of the fast wind, the presence of Alfvénic turbulence causes the heavier ions to flow faster than protons by a sizable fraction of the Alfvén speed. Although this fraction is similar to that observed at $1 \mathrm{AU}$, the Alfvén speed at $0.3 \mathrm{AU}$ is approximately a factor 3 larger than at Earth. Similarly, the heavier ion temperatures are markedly larger in the fast solar wind than those of the protons.

[55] The fast solar wind appears to be a blend of thermal speeds that are determined in the heliosphere and those determined in the solar corona. The ratios of ion temperatures to proton temperatures at $\sim 0.3 \mathrm{AU}$ are larger than would result if the ions simply acquired a fraction of the Alfvén speed as their thermal speed, as is observed at 1 AU, providing evidence of $\mathrm{m} / \mathrm{q}$-dependent heating that has been observed spectroscopically at the Sun. An examination of the magnetic environment at both MESSENGER and ACE reinforces this observation, as although the turbulent power associated with Alfvénic fluctuations is an order of magnitude higher at $R \sim 0.3 \mathrm{AU}$ than at Earth, there is no strong 
spectral signature of local heating at either heliocentric distance.

[56] Despite the fact that the FIPS sensor on MESSENGER, with its view partially obstructed by the spacecraft sunshade, is not ideally suited for observing the solar wind, it has been possible to draw conclusions about the properties of heavier ions in the solar wind at $0.3 \mathrm{AU}$, and thus place constraints on the mechanisms of the acceleration and evolution of the solar wind as it propagates outward from the Sun. Moreover, we should expect that the accuracy of the results presented here will improve with additional observations and sharpened statistics as MESSENGER continues in its extended mission.

\section{Appendix A: Moments of Partial Velocity Distribution Functions}

[57] To understand the effects of observing only a fraction of the solar wind plasma, we take the moments of the obstructed drifting isotropic Maxwell-Boltzmann velocity distribution defined by

$$
f\left(v_{\mathrm{x}}, v_{\mathrm{y}}, v_{\mathrm{z}}\right)=n_{\mathrm{o}}\left(\frac{1}{2 \pi}\right)^{3 / 2} \frac{1}{v_{\mathrm{th}}^{3}} \exp \left(-\frac{\left(\left(v_{\mathrm{x}}-v_{\mathrm{o}}\right)^{2}+v_{\mathrm{y}}^{2}+v_{\mathrm{z}}^{2}\right)}{2 v_{\mathrm{th}}^{2}}\right) .
$$

[58] Here, $n_{\mathrm{o}}$ is the proton density, $v_{\mathrm{o}}$ is the bulk speed, and $v_{\text {th }}$ is the thermal speed that is related to the proton temperature by the definition

$$
v_{\text {th }} \equiv \sqrt{\frac{k_{\mathrm{B}} T}{m}}
$$

[59] The plasma will be visible for all $v_{\mathrm{x}}$ and $v_{\mathrm{y}}$ but will be obstructed in the $v_{\mathrm{z}}$ direction for $v_{\mathrm{z}}<v_{\mathrm{z}, \min }$, as shown in Figure A1. It is assumed that no more than $50 \%$ of the distribution function will be in the observable range $v_{z, \min }>0$. The distribution function in Figure A1 is not in the same references frame as that illustrated in Figure 2. The frame center here was shifted to a lower $v_{\mathrm{z}}$ value by $v_{\mathrm{z}, \mathrm{min}}$ to provide a mathematically simplified analysis that can then related to more detailed instrument geometry.

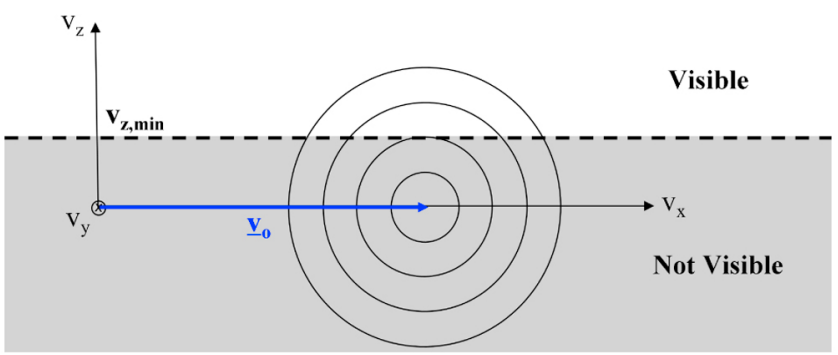

Figure A1. Illustration of an obstructed isotropic drifting Maxwell-Boltzmann velocity distribution with center $\left[v_{\mathrm{o}}, 0,0\right]$. The distribution is visible for all values of $v_{\mathrm{x}}$ and $v_{\mathrm{y}}$ but blocked for $v_{\mathrm{z}}<v_{\mathrm{z} \text {,min }}$.
[60] The observed moments of interest are the 0th (density), 1st (velocity), and 2nd (temperature) moments of the distribution function, defined here as

$$
\begin{gathered}
n_{\mathrm{obs}}=\iiint d v_{\mathrm{z}} d v_{\mathrm{y}} d v_{\mathrm{x}} \cdot f\left(v_{\mathrm{x}}, v_{\mathrm{y}}, v_{z}\right) \\
n_{\mathrm{obs}}\langle\mathbf{v}\rangle=\iiint d v_{\mathrm{z}} d v_{\mathrm{y}} d v_{\mathrm{x}} \cdot \mathbf{v} \cdot f\left(v_{\mathrm{x}}, v_{\mathrm{y}}, v_{\mathrm{z}}\right) \text {, and } \\
n_{\mathrm{obs}\left\langle\left(\mathbf{v}-\mathbf{v}_{\mathrm{o}}\right)^{2}\right\rangle}=\iiint d v_{\mathrm{z}} d v_{\mathrm{y}} d v_{\mathrm{x}} \cdot\left(\mathbf{v}-\mathbf{v}_{\mathrm{o}}\right)^{2} \cdot f\left(v_{\mathrm{x}}, v_{\mathrm{y}}, v_{\mathrm{z}}\right) .
\end{gathered}
$$

[61] To take into account the obstruction, the limits of integration in (A3)-(A5) are from $-\infty$ to $+\infty$ for $v_{\mathrm{x}}$ and $v_{\mathrm{y}}$, and for $v_{\mathrm{z}}$ are from $v_{\mathrm{z}, \mathrm{min}}$ to $+\infty$.

\section{A1. Density}

[62] Integrating (A3) yields

$$
\begin{aligned}
n_{\mathrm{obs}} & =\int_{v_{z, \min }}^{\infty} d v_{\mathrm{z}} \int_{-\infty}^{\infty} d v_{\mathrm{y}} \int_{-\infty}^{\infty} d v_{\mathrm{x}} \cdot f\left(v_{\mathrm{x}}, v_{\mathrm{y}}, v_{\mathrm{z}}\right) \\
& =\frac{n_{\mathrm{o}}}{2} \cdot \operatorname{erfc}\left(\frac{v_{\mathrm{z}, \text { min }}}{v_{\text {th }}}\right) .
\end{aligned}
$$

where erfc is the complementary error function. Because only a partial distribution function is observed, only a fraction of the density is recovered, i.e., $n_{\mathrm{obs}}<n_{\mathrm{o}} / 2$. Unless $v_{\mathrm{z}, \mathrm{min}} / v_{\text {th }}$ is well known, the total density $n_{\mathrm{o}}$ cannot be determined. Therefore, for an observing instrument, unless the location of the core of the distribution is well constrained, it will be difficult to recover the density from a partially observed distribution.

\section{A2. Velocity}

[63] Integrating (A4) yields,

$\left\langle v_{\mathrm{x}}\right\rangle=v_{\mathrm{o}}$,

$\left\langle v_{\mathrm{y}}\right\rangle=0$, and

$\left\langle v_{\mathrm{z}}\right\rangle=\frac{\iiint v_{\mathrm{z}} \cdot d v_{\mathrm{z}} d v_{\mathrm{y}} d v_{\mathrm{x}} \cdot f\left(v_{\mathrm{x}}, v_{\mathrm{y}}, v_{\mathrm{z}}\right)}{n_{\mathrm{obs}}}$

$$
\begin{aligned}
& =\sqrt{\frac{2}{\pi}} \cdot v_{\text {th }} \cdot \frac{\exp \left(\frac{-v_{z, \min }^{2}}{2 v_{\text {th }}^{2}}\right)}{\operatorname{erfc}\left(\frac{v_{z, \text { min }}}{v_{\text {th }} \sqrt{2}}\right)} . \\
& \approx \begin{cases}\sqrt{\frac{2}{\pi}} \cdot v_{\text {th }}, & \frac{v_{z, \text { min }}}{v_{\text {th }}} \ll 1 \\
v_{z, \text { min }}, & \frac{v_{z, \text { min }}}{v_{\text {th }}} \gg 1 .\end{cases}
\end{aligned}
$$


[64] Unlike $\left\langle v_{\mathrm{y}}\right\rangle,\left\langle v_{\mathrm{z}}\right\rangle$ will yield a non-zero value as a consequence of its non-symmetric limits of integration. Two limiting cases of $\left\langle v_{z}\right\rangle$ can be considered here and are shown in equation (A10). For $v_{\mathrm{z}, \mathrm{min}} / v_{\text {th }} \ll 1$, i.e., if approximately $50 \%$ of the distribution is visible, $\left\langle v_{z}\right\rangle$ is offset from the distribution center by approximately $v_{\text {th }}$. For $v_{z, \min } / v_{\text {th }} \gg 1$, $\left\langle v_{z}\right\rangle$ will be equal to $v_{z \text {,min }}$, i.e., the location of the obstruction. For either case, for a distribution with a bulk velocity $v_{\mathrm{o}} / v_{\mathrm{th}}, \mathrm{v}_{\mathrm{o}} / v_{\mathrm{z}, \min } \gg 1$, the error introduced into the determination of $\langle v\rangle$ from a $\left\langle v_{z}\right\rangle$ offset will be relatively small.

\section{A3. Temperature}

[65] For $v_{\mathrm{o}} / v_{\mathrm{th}}, v_{\mathrm{o}} / v_{z, \min } \gg 1$, integrating (A5) yields,

$$
\begin{aligned}
\left\langle\left(\mathbf{v}-\mathbf{v}_{\mathrm{o}}\right)^{2}\right\rangle & \approx\left\langle\left(v_{\mathrm{x}}-v_{\mathrm{o}}\right)^{2}\right\rangle \\
& =\frac{\iiint d v_{\mathrm{z}} d v_{\mathrm{y}} d v_{\mathrm{x}} \cdot\left(v_{\mathrm{x}}-v_{\mathrm{o}}\right)^{2} \cdot f\left(v_{\mathrm{x}}, v_{\mathrm{y}}, v_{\mathrm{z}}\right)}{n_{\mathrm{obs}}}=v_{\mathrm{th}}^{2} .
\end{aligned}
$$

[66] For a supersonic plasma, the thermal velocity can therefore be recovered independent of the fraction of the distribution that is visible. The recovered temperature from an isotropic Maxwellian velocity distribution is therefore expected to be accurate provided it is above the detection limit.

\section{Appendix B: Derivation of Observed Distribution Function}

[67] Equation (1) can be substituted into (2), and the Bessel function identity, $I_{\mathrm{o}}(x)=(1 / 2 \pi) \int_{0}^{2 \pi} d \phi \cdot \exp (x \cos \phi)$ [Arfken et al., 2000], where $I_{\mathrm{o}}$ is modified Bessel function of the first kind, can be used to replace the integration over $\phi$, yielding

$$
\begin{aligned}
f_{\text {obs }}(v)= & \int_{0}^{\pi / 2} \sin \theta d \theta \cdot n_{\mathrm{o}}\left(\frac{1}{2 \pi}\right)^{3 / 2} \frac{1}{v_{\text {th }}^{3}} \\
& \cdot \exp \left(-\frac{v^{2}+v_{\mathrm{o}}^{2}-2 v v_{\mathrm{o}} \cos \theta \cos \theta_{\mathrm{o}}}{2 v_{\mathrm{th}}^{2}}\right) \\
& \times 2 \pi \cdot I_{\mathrm{o}}\left(\frac{v v_{\mathrm{o}} \sin \theta \sin \theta_{\mathrm{o}}}{v_{\mathrm{th}}^{2}}\right) .
\end{aligned}
$$

[68] For a supersonic distribution, i.e., $v_{\mathrm{o}} / v_{\text {th }} \gg 1$, and using the asymptotic approximation, $I_{\mathrm{o}}(x) \approx \exp (x) / \sqrt{2 \pi x}$, $x \gg 1 / 4$, (B1) can be written as

$$
\begin{aligned}
f_{\mathrm{obs}}(v)= & n_{\mathrm{o}} \cdot \frac{1}{2 \pi} \frac{1}{v_{\mathrm{th}}^{2} \sqrt{v v_{\mathrm{o}}}} \int_{0}^{\pi / 2} d \theta \cdot \sqrt{\frac{\sin \theta}{\sin \theta_{\mathrm{o}}}} \\
& \times \exp \left(-\frac{v^{2}+v_{\mathrm{o}}^{2}-2 v v_{\mathrm{o}} \cos \left(\theta-\theta_{\mathrm{o}}\right)}{2 v_{\mathrm{th}}^{2}}\right) .
\end{aligned}
$$

[69] Since $\theta \approx \theta_{o} \approx \pi / 2$ for any expected solar wind distribution, equation (B2) can be approximated as

$$
\begin{aligned}
& f_{\text {obs }}(v) \approx n_{\mathrm{o}} \\
& \cdot \frac{1}{2 \pi} \frac{1}{v_{\mathrm{th}}^{2} \sqrt{v v_{\mathrm{o}}}} \exp \left(-\frac{\left(v-v_{\mathrm{o}}\right)^{2}}{2 v_{\mathrm{th}}^{2}}\right) \\
& \times \int_{0}^{\pi / 2} d \theta \cdot \sqrt{\frac{\sin \theta}{\sin \theta_{\mathrm{o}}}} \exp \left(-\frac{v v_{\mathrm{o}}}{2 v_{\mathrm{th}}^{2}}\left(\theta-\theta_{\mathrm{o}}\right)^{2}\right) .
\end{aligned}
$$

[70] For $\sqrt{\frac{\sin \theta}{\sin \theta_{\mathrm{o}}}} \approx 1$, equation (B3) can be integrated analytically, yielding

$$
f_{\mathrm{obs}}(v) \approx n_{\mathrm{o}} \cdot D \cdot \frac{1}{\sqrt{2 \pi} v v_{\mathrm{o}} v_{\mathrm{th}}} \exp \left(-\frac{\left(v-v_{\mathrm{o}}\right)^{2}}{2 v_{\mathrm{th}}^{2}}\right),
$$

where

$$
D=\frac{1}{2} \operatorname{erfc}\left(\sqrt{\frac{v v_{\mathrm{o}}}{2 v_{\mathrm{th}}^{2}}}\left(\theta_{\mathrm{o}}-\frac{\pi}{2}\right)\right) \approx \frac{1}{2} \operatorname{erfc}\left(\frac{v_{\mathrm{o}}}{v_{\mathrm{th}} \sqrt{2}}\left(\theta_{\mathrm{o}}-\frac{\pi}{2}\right)\right)
$$

[71] Equation (B5) is in the form of the observed distribution function described by von Steiger et al. [2000] for Ulysses/SWICS, but with a different effective duty cycle factor, $D$. $D$ depends on both $v$ and $\theta_{\mathrm{o}}$. However, for a supersonic distribution, $D$ is assumed to be constant in order to derive analytical moments of $f_{\text {obs }}$.

[72] Acknowledgments. The MESSENGER project is supported by the NASA Discovery Program under contracts NAS5-97271 to The Johns Hopkins University Applied Physics Laboratory and NASW-00002 to the Carnegie Institution of Washington. This work was also supported by the NASA Graduate Student Research Program grant NNX09AL50H (DJG), and the National Science Foundation grant AGS-1043012 (LAF).

[73] Philippa Browning thanks Rudolf von Steiger and another reviewer for their assistance in evaluating the paper.

\section{References}

Anderson, B. J., M. H. Acuña, D. A. Lohr, J. Scheifele, A. Raval, H. Korth, and J. A. Slavin (2007), The Magnetometer instrument on MESSENGER, Space Sci. Rev., 131, 417-450.

Andrews, G. B., et al. (2007), The Energetic Particle and Plasma Spectrometer instrument on the MESSENGER spacecraft, Space Sci. Rev., 131, 523-556.

Arfken, G. B., H. J. Weber, and F. Harris (2000), Mathematical Methods for Physicists, 5th ed., 1182 pp., Elsevier, Amsterdam.

Asbridge, J. R., S. J. Bame, W. C. Feldman, and M. D. Montgomery (1976), Helium and hydrogen velocity differences in the solar wind, J. Geophys. Res., 81, 2719-2727.

Bale, S. D., P. J. Kellogg, F. S. Mozer, T. S. Horbury, and H. Reme (2005), Measurement of the electric fluctuation spectrum of magnetohydrodynamic turbulence, Phys. Rev. Lett., 94, 215002.

Bavassano, B., M. Dobrowolny, F. Mariani, and N. F. Ness (1982), Radial evolution of power spectra of interplanetary Alfvénic turbulence, J. Geophys. Res., 87, 3617-3622.

Belcher, J. W., and L. J. Davis (1971), Large-amplitude Alfvén waves in the interplanetary medium: 2, J. Geophys. Res., 76, 3534-3563.

Berger, L., R. F. Wimmer-Schweingruber, and G. Gloeckler (2011), Systematic measurements of ion-proton differential streaming in the solar wind, Phys. Rev. Lett., 106, 151103.

Bochsler, P., et al. (2010), Kinetic temperatures of iron ions in the solar wind observed with STEREO/PLASTIC, in Twelfth International Solar Wind Conference, edited by M. Maksimovic et al., AIP Conf. Proc., 1216, 257-260, doi:10.1063/1.3395850.

Coleman, P. J. (1968), Turbulence, viscosity, and dissipation in thesolarwind plasma, Astrophys. J., 153, 371-388. 
Cranmer, S. R., A. A. van Ballegooijen, and R. J. Edgar (2007), Selfconsistent coronal heating and solar wind acceleration from anisotropic magnetohydrodynamic turbulence, Astrophys. J. Suppl. Ser., 171, 520-551.

Fisk, L. A., and G. Gloeckler (2006), The common spectrum for accelerated ions in the quiet-time solar wind, Astrophys. J., 640, L79-L82.

Fisk, L. A., N. A. Schwadron, and T. H. Zurbuchen (1999), Acceleration of the fast solar wind by the emergence of new magnetic flux, J. Geophys. Res., 104, 19,765-19,772.

Fisk, L. A., G. Gloeckler, and N. A. Schwadron (2010), On theories for stochastic acceleration in the solar wind, Astrophys. J., 720, 533-540.

Freeman, J. W. (1988), Estimates of solar wind heating inside 0.3 AU, Geophys. Res. Lett., 15, 88-91.

Frisch, U. (1996), Turbulence, 312 pp., Cambridge Univ. Press, Cambridge, U. K.

Galvin, A. B., et al. (2008), The Plasma and Suprathermal Ion Composition (PLASTIC) investigation on the STEREO observatories, Space Sci. Rev. 136, 437-486.

Gary, S. P., B. E. Goldstein, and J. T. Steinberg (2001), Helium ion acceleration and heating by Alfvén/cyclotron fluctuations in the solar wind J. Geophys. Res., 106, 24,955-24,963.

Geiss, J., P. Hirt, and H. Leutwyler (1970), On acceleration and motion of ions in corona and solar wind, Sol. Phys., 12, 458-483.

Gloeckler, G., et al. (1992), The solar wind ion composition spectrometer, Astron. Astrophys. Suppl., 92, 267-289.

Gloeckler, G., et al. (1998), Investigation of the composition of solar and interstellar matter using solar wind and pickup ion measurements with SWICS and SWIMS on the ACE spacecraft, Space Sci. Rev., 86, 497-539.

Goldstein, B. E., M. Neugebauer, and E. J. Smith (1995), Alfvén waves, alpha particles, and pickup ions in the solar wind, Geophys. Res. Lett., 22, 3389-3392.

Goldstein, M. L., D. A. Roberts, and W. H. Matthaeus (1995), Magnetohydrodynamic turbulence in the solar wind, Annu. Rev. Astron. Astrophys., $33,283-326$.

Gosling, J. T., D. J. McComas, D. A. Roberts, and R. M. Skoug (2009), A one-sided aspect of Alfvénic fluctuations in the solar wind, Astrophys. J. Lett., 695, L213-L216.

Grappin, R., A. Mangeney, and E. Marsch (1990), On the origin of solar wind MHD turbulence: Helios data revisited, J. Geophys. Res., 95 , 8197-8209.

Hamilton, K., C. W. Smith, B. J. Vasquez, and R. J. Leamon (2008), Anisotropies and helicities in the solar wind inertial and dissipation ranges at 1 AU, J. Geophys. Res., 113, A01106, doi:10.1029/2007JA012559.

Hefti, S., et al. (1998), Kinetic properties of solar wind minor ions and protons measured with SOHO/CELIAS, J. Geophys. Res., 103, $29,697-29,704$

Hundhausen, A. J., S. J. Bame, J. R. Asbridge, and S. J. Sydoriak (1970), Solar wind proton properties: Vela 3 observations from July 1965 to June 1967, J. Geophys. Res., 75, 4643-4657.

Isenberg, P. A., and J. V. Hollweg (1982), Finite amplitude Alfvén waves in a multi-ion plasma: Propagation, acceleration, and heating, J. Geophys. Res., 87, 5023-5029.

Kasper, J. C., A. J. Lazarus, and S. P. Gary (2008), Hot solar-wind helium: Direct evidence for local heating by Alfvén-cyclotron dissipation, Phys. Rev. Lett., 101, 261103, doi:10.1103/PhysRevLett.101.261103.

Kohl, J. L., et al. (1995), The ultraviolet coronagraph spectrometer for the Solar and Heliospheric Observatory, Sol. Phys., 162, 313-356.

Kohl, J. L., et al. (1997), First results from the SOHO ultraviolet coronagraph spectrometer, Sol. Phys., 175, 613-644.

Kohl, J. L., et al. (1998), UVCS/SOHO empirical determinations of anisotropic velocity distributions in the solar corona, Astrophys. J. Lett., 501, L127-L131, doi:10.1086/311434.

Leamon, R. J., C. W. Smith, N. F. Ness, W. H. Matthaeus, and H. K. Wong (1998), Observational constraints on the dynamics of the interplanetary magnetic field dissipation range, J. Geophys. Res., 103, 4775-4787.

Leamon, R. J., C. W. Smith, N. F. Ness, and H. K. Wong (1999), Dissipation range dynamics: Kinetic Alfvén waves and the importance of $\beta_{\mathrm{e}}$, J. Geophys. Res., 104, 22,331-22,344.
Li, X. (1999), Proton temperature anisotropy in the fast solar wind: A 16moment Bi-Maxwellian model, J. Geophys. Res., 104, 19,773-19,786.

Marsch, E. (1991), Turbulence in the solar wind, in Reviews in Modern Astronomy, vol. 4, edited by G. Klare, pp. 145-156, Springer, Berlin.

Marsch, E., K. H. Mühlhäuser, H. Rosenbauer, R. Schwenn, and F. M. Neubauer (1982a), Solar wind helium ions: Observations of the Helios solar probes between 0.3 and 1 AU, J. Geophys. Res., 87, 35-51.

Marsch, E., K. H. Mühlhäuser, R. Schwenn, H. Rosenbauer, W. Pilipp, and F. M. Neubauer (1982b), Solar wind protons: Three-dimensional velocity distributions and derived plasma parameters measured between 0.3 and 1 AU, J. Geophys. Res., 87, 52-72.

McComas, D. J., S. J. Bame, P. Barker, W. C. Feldman, J. L. Phillips, P. Riley, and J. W. Griffee (1998a), Solar wind electron proton alpha monitor (SWEPAM) for the Advanced Composition Explorer, Space Sci. Rev., 86, 563-612.

McComas, D. J., et al. (1998b), Ulysses' return to the slow solar wind, Geophys. Res. Lett., 25, 1-4.

McKenzie, J. F., M. Banaszkiewicz, and W. I. Axford (1995), Acceleration of the high speed solar wind, Astron. and Astrophys., 303, L45-L48.

Neugebauer, M. (1976), The role of Coulomb collisions in limiting differential flow and temperature differences in the solar wind, J. Geophys. Res., 81, 78-82.

Ogilvie, K. W., et al. (1995), SWE, a comprehensive plasma instrument for the WIND spacecraft, Space Sci. Rev., 71, 55-77.

Parker, E. N. (1958), Dynamics of the interplanetary gas and magnetic fields, Astrophys. J., 128, 664-676.

Roberts, D. A., M. L. Goldstein, L. W. Klein, and W. H. Matthaeus (1987) Origin and evolution of fluctuations in the solar wind: Helios observations and Helios-Voyager comparisons, J. Geophys. Res., 92,12,023-12,035.

Rosenbauer, H., et al. (1977), A survey on initial results of the HELIOS plasma experiment, J. Geophys. Res., 42, 561-580.

Schwenn, R., H. Rosenbauer, and H. Miggenrieder (1975), The plasma experiment on board Helios/E 1, Raumfahrtforschung, 19, 226-232.

Smith, C. W., J. L'Heureux, N. F. Ness, M. H. Acuña, L. F. Burlaga, and J. Scheifele (1998), The ACE magnetic fields experiment, Space Sci. Rev., 86, 613-632.

Smith, C. W., B. J. Vasquez, and J. V. Hollweg (2012), Observational constraints on the role of cyclotron damping and kinetic Alfvén waves in the solar wind, Astrophys. J., 745, 1-8.

Smith, E. J., A. Balogh, M. Neugebauer, and D. McComas (1995), Ulysses observations of Alfvén waves in the southern and northern solar hemispheres, Geophys. Res. Lett., 22, 3381-3384.

Solomon, S. C., et al. (2001), The MESSENGER mission to Mercury: Scientific objectives and implementation, Planet. Space Sci., 49 , 1445-1465, doi:10.1016/S0032-0633(01)00085-X.

Totten, T. L., J. W. Freeman, and S. Arya (1995), An empirical determination of the polytropic index for the free-streaming solar wind using Helios 1 data, J. Geophys. Res., 93, 13-17.

Vasyliunas, V. M. (1968), A survey of low-energy electrons in the evening sector of the magnetosphere with OGO 1 and OGO 3, J. Geophys. Res., 73, 2839-2884.

Verdini, A., and M. Velli (2007), Alfvén waves and turbulence in the solar atmosphere and solar wind, Astrophys. J., 662, 669-676.

von Steiger, R., and T. H. Zurbuchen (2006), Kinetic properties of heavy solar wind ions from Ulysses-SWICS, Geophys. Res. Lett., 33, L09103, doi:10.1029/2005GL024998.

von Steiger, R., N. A. Schwadron, L. A. Fisk, J. Geiss, G. Gloeckler, S. Hefti, B. Wilken, R. R. Wimmer-Schweingruber, and T. H. Zurbuchen (2000), Composition of quasi-stationary solar wind flows from Ulysses/ Solar Wind Ion Composition Spectrometer, J. Geophys. Res., 105, 27,217-27,238

Wolfe, J. H., R. W. Silva, D. D. McKibbin, and R. H. Mason (1966), The compositional, anisotropic, and nonradial flow characteristics of the solar wind, J. Geophys. Res., 71, 3329-3335.

Zurbuchen, T. H., L. A. Fisk, N. A. Schwadron, and G. Gloeckler (2000), Observations of non-thermal properties of heavy ions in the solar wind, in Acceleration and Transport of Energetic Particles Observed in the Heliosphere, edited by R. A. Mewaldt et al., AIP Conf. Proc., 528, 215-220. 